\title{
A HIGMAN EMBEDDING PRESERVING ASPHERICITY
}

\author{
MARK SAPIR
}

\section{Contents}

1. Introduction

2. Preliminaries

2.1. Van Kampen diagrams

2.2. Asphericity and free constructions

3. An auxiliary group

4. The main construction

4.1. Preliminaries on $S$-machines 22

4.2. Four $S$-machines 26

4.3. The composition of the $S$-machines and the auxiliary group $\quad 28$

4.4. The group $\mathcal{G}$

5. The main result

5.1. The map $\varepsilon$ is a homomorphism

5.2. Expanded presentation of $\mathcal{G}$

5.3. Sectors 36

5.4. Diagrams with boundary label over $\varepsilon(A)$

Acknowledgements

References

\section{INTRODUCTION}

The main result of this paper is the following theorem (see Theorem 5.1). For definitions see Section 2.2

Theorem 1.1. Every finitely generated group with combinatorially aspherical recursive presentation complex embeds into a group with finite combinatorially aspherical presentation complex.

Using Davis's construction [8] (see also 9, Chapter 11]), this allows one to create closed aspherical manifolds of dimension 4 and higher with some previously un-

Received by the editors April 26, 2011 and, in revised form, September 22, 2011, November 29, 2011, and April 29, 2013.

2010 Mathematics Subject Classification. Primary 20F65; Secondary 20F69, 20F38, 22 F50.

Key words and phrases. Aspherical group, Higman embedding, S-machine.

This research was supported in part by NSF grant DMS-0700811.

(C) 2013 American Mathematical Society Reverts to public domain 28 years from publication 
known "extreme" properties1 1 For example, by Gromov [12 (for more details see Arzhantseva and Delzant [1] and for a completely self-contained proof see Coulon [7]), there exists a finitely generated group with recursive combinatorially aspherical presentation whose Cayley graph coarsely contains an expander. Hence Theorem 1.1 implies

Corollary 1.2. There exist closed aspherical manifolds of dimension 4 and higher whose fundamental groups coarsely contain expanders.

The fundamental groups of the manifolds from Corollary 1.2 are not coarsely embeddable into a Hilbert space [12, do not satisfy G. Yu's property A [32, and are counterexamples to the Baum-Connes conjecture with coefficients (see Higson, Lafforgue, Skandalis [16). They also have infinite asymptotic dimension. This solves a problem, formulated first by G. Yu in [31, asking whether the fundamental group of a closed aspherical manifold can have infinite asymptotic dimension. A weaker problem of whether the asymptotic dimension of a closed aspherical manifold can exceed its (ordinary) dimension was mentioned by Gromov in [11, p. 33] and remained open until now. Note that Dranishnikov's problem of whether the asymptotic dimension of an aspherical $n$-manifold is always $n$ or infinity is still open (see [10, Problem 3.4]). It seems that Gromov's random groups and our Theorem 1.1 cannot give an example of where the dimensions are different while both are finite, in view of the recent paper by Willett 30$]^{2}$

As another corollary one can deduce that

Corollary 1.3. A torsion-free Tarski monster (that is, a finitely generated group all of whose proper subgroups are infinite cyclic; see Ol'shanskiu [20]) embeds into the fundamental group of a closed aspherical manifold.

Indeed, by 20, the torsion-free Tarski monsters constructed by Ol'shanskiu have recursive combinatorially aspherical presentations; it remains to apply Theorem 1.1 and 8 . More generally, every lacunary hyperbolic group given by a recursive graded small cancelation presentation (see 22) embeds into the fundamental group of a closed aspherical manifold.

Note the fact that any recursively presented group that embeds into a finitely presented group is the celebrated Higman embedding theorem [15], which is one of the main results in algorithmic group theory. There exist at least ten versions of Higman's embedding construction preserving various properties of groups, from relatively easy constructions to very complicated (see [25], 3], 22, 23], 26], 27] and references therein). But all the previous versions could not produce finitely presented groups with finite $K(., 1)$ if the original group was not finitely presented. The reason is also basically common to all these constructions and can be roughly described as follows. Let $G$ be a finitely presented group containing a copy of a recursively presented group $\Gamma$ and constructed using one of the existing proofs of the Higman embedding theorem. Then in most known constructions there exists $1 \neq g \in G$ which centralizes $\Gamma$. Consider two copies $\Delta_{1}, \Delta_{2}$ of a van Kampen diagram over the presentation of $G$ with boundaries labeled by a relation $r$ of $\Gamma$ (such diagrams must exist by the van Kampen lemma since $r=1$ in $G$ ). Consider

\footnotetext{
${ }^{1}$ As explained in [9, Chapter 11], in dimension 5 and higher, one can assume that the aspherical manifold we construct is smooth. This was communicated to the author by I. Belegradek.

${ }^{2}$ For a recent survey about aspherical manifolds see Lück [17]; for constructions of aspherical manifolds with other "exotic" properties see Davis [9].
} 


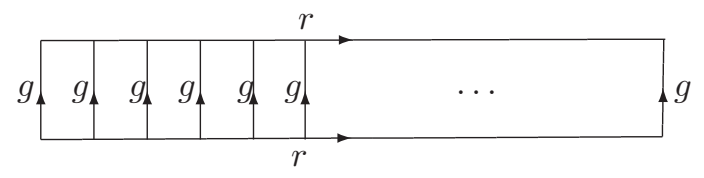

Figure 1

the van Kampen diagram $\Delta_{3}$ for the commutativity relation $g r=r g$ obtained by gluing together diagrams for $g a=a g$ for every letter $a$ in $r$ (see Figure 1). It has the form of a rectangle with sides labeled by $r, g, r^{-1}, g^{-1}$. Glue $\Delta_{1}, \Delta_{2}, \Delta_{3}$ together to form a spherical van Kampen diagram over the presentation of $G$. That spherical diagram is not combinatorially homotopic to a trivial diagram. Moreover it is basically clear that if the defining relations of $\Gamma$ are independent enough (say, satisfy some form of small cancelation as in many interesting cases), then these spherical diagrams cannot be "generated" by finitely many spherical diagrams over the presentation of $G$. Hence the relation module [18 of the group $G$ is infinitely generated. A similar problem arises even if we assume only $g \Gamma g^{-1} \subset \Gamma$. More complicated constructions such as the one from [23] have the same problem but it is hidden deeper.

The main difficulty that we had to overcome in this paper was to avoid this type of "trivial" spherical diagrams. In our construction, we are using $S$-machines (which can be viewed as multiple HNN-extensions of free groups) first introduced in [29] and used for some versions of Higman embedding in 2], 23] and other papers. The finitely presented group in this paper is built from two (different) $S$-machines and several hyperbolic and close to hyperbolic groups that "glue" these $S$-machines together. One of the main tools of the proof is the congruence extension property of certain subgroups of hyperbolic groups first established by Ol'shanskil in [21.

One can view this paper as giving examples of finitely presented groups with extreme properties. There are now many examples of finitely generated extreme groups, sometimes called monsters, but finitely presented monsters are much more rare. There are some objective reasons for this. For example a hyperbolic group version of the Cartan-Hadamard theorem proved by Gromov (see the Appendix of 22. by Kapovich and Kleiner) shows that a lacunary hyperbolic finitely presented group is hyperbolic and so it cannot be torsion, have few subgroups, or be of intermediate growth, etc. Since many methods of creating extreme groups are based on lacunar hyperbolicity [20], 22], these methods cannot produce finitely presented groups.

\section{Preliminaries}

2.1. Van Kampen diagrams. The main tool in this paper is van Kampen diagrams (18, 20]). For the benefit of the reader, in this section, we present an overview of van Kampen diagrams and show how van Kampen diagrams can be used.

2.1.1. The definition. Let $G$ be a finitely presented group given by a finite set of generators $X$ and a finite set of relators $R$, let $\phi$ be a homomorphism from the free group $F(X)$ onto $G$, and let $N$ be the kernel of this homomorphism.

Since $N$ is generated by $R$ as a normal subgroup, a reduced group word $W$ from $F(X)$ is in $N$ if and only if $W$ can be represented in the free group as a product of 
conjugates of elements of $R$ and their inverses:

$$
W=\prod_{i=1}^{m} s_{i} r_{i}^{ \pm 1} s_{i}^{-1}
$$

where $s_{i} \in F(X), r_{i} \in R$.

For every representation (2.1), we can draw a bouquet of "lollipops" which is a planar labeled graph. Each "lollipop" corresponds to one of the factors $s_{i} r_{i} s_{i}^{-1}$, it has a stem, a path labeled by $s_{i}$ (i.e. the stem is subdivided into edges labeled by the letters of $s_{i}$ ), and a candy, a cycle path labeled by $r_{i}$ (see Figure 2).
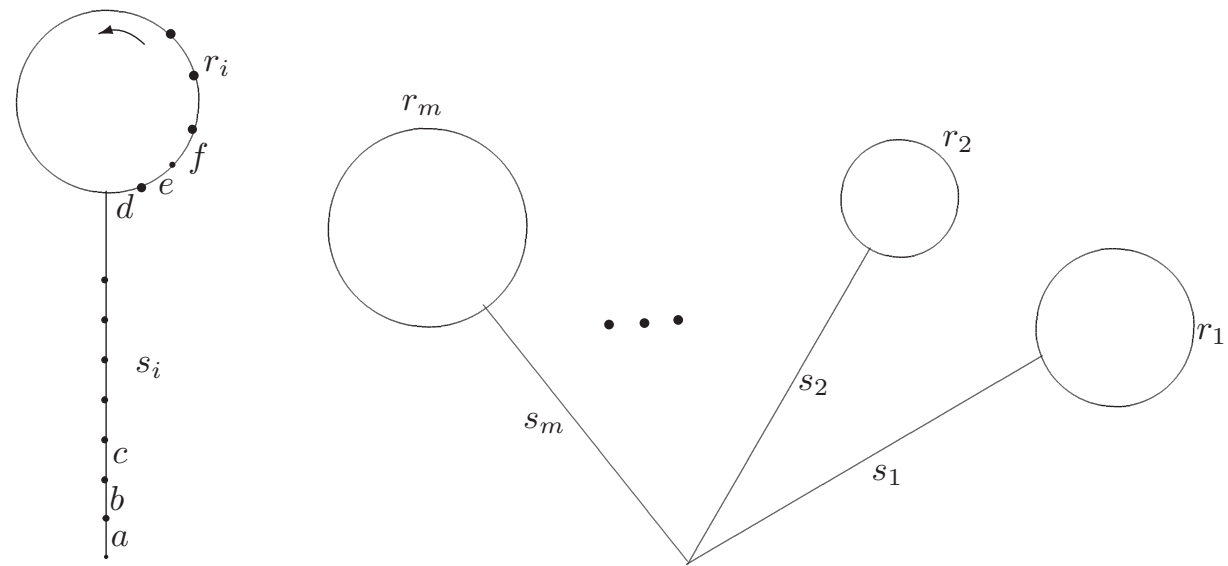

FiguRE 2. Using lollipops to build a van Kampen diagram.

Going counterclockwise around the "lollipop" starting and ending at the tip of the stem, we read $s_{i} r_{i} s_{i}^{-1}$. Thus going counterclockwise around the diagram which is the bouquet of "lollipops", we read the word which is the right-hand side of (2.1).

In order to make the word $W$ from this word, we need to reduce the boundary of the bouquet of "lollipops" (the boundary is traced counterclockwise): every time we see a pair of consecutive edges on the boundary of the diagram, which have the same label and the same initial or terminal vertex (see Figure 3), we identify these two edges (if the edges have both vertices in common, we identify the two edges and remove the whole subgraph bounded by them on the plane). This amounts to removing subwords $x x^{-1}$ and $x^{-1} x$ from the right-hand side of (2.1). The resulting picture is a van Kampen diagram for $W$ over the presentation $\langle X \mid R\rangle$, that is, a planar graph with edges labeled by elements of $X$, the boundary of each cell (i.e. the closure of a bounded connected components of the plane minus the graph) labeled by words from $R^{ \pm 1}$, and the boundary of the whole graph (i.e. the boundary of the infinite component of the plane minus the graph) is labeled by $W$ (see 18 for a slightly different definition and 20 for another one). 

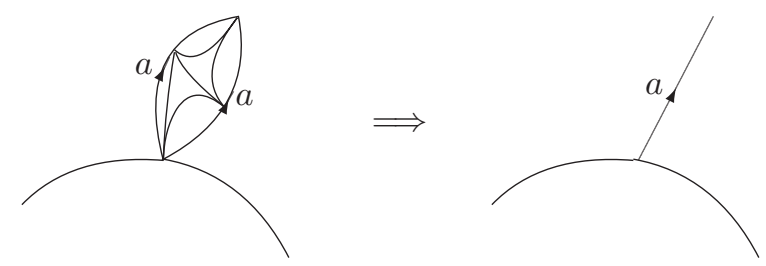

FiguRE 3. Reducing the boundary.

Figure 4 shows what a typical van Kampen diagram may look like. One can see that the cells may be of different sizes and shapes, a cell can touch itself, etc. It is also important that a diagram itself may not be an embedded disc: several pieces as in Figure 4 can be connected by paths to form a tree of discs (as the bouquet of lollipops in Figure 2).

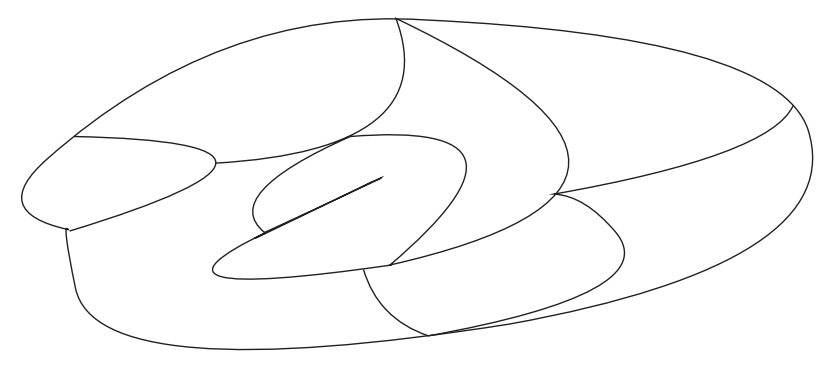

Figure 4. A van Kampen diagram without labels.

The main (but simple) fact about van Kampen diagrams is the following

Lemma 2.1 (Van Kampen lemma [18, 20, 28). A reduced group word $W$ over the alphabet $X$ is equal to 1 in $G$ if and only if there exists a van Kampen diagram over the presentation of $G$ with boundary label $W$.

2.1.2. 0-cells. Following Ol'shanskiu [20, we are going to use 0-cells. A 0-edge is an edge labeled by 1 (the identity element of the group). A 0-relation is a relation of one of two forms $1^{k}=1$ and $1^{k} a 1^{l} a^{-1} 1^{m}=1, k, l, m \geq 0$. A cell corresponding to a 0 -relation is called a 0 -cell; see Figure 5 ,

A 0 -cell is a cell corresponding to a 0-relation. Clearly, adding 0-relations to a presentation does not change the group. 0-cells allow us to assume that every cell in a diagram and the diagram itself are embedded discs. For example, Figures 6 and 7 show what to do when a cell touches itself and when a diagram consists of two disc diagrams connected by an arc.

As noted in 20, Chapter 4], using 0-cells, one can transform every van Kampen diagram $\Delta$ into a diagram $\Delta^{\prime}$ over the "extended presentation" (including the 0 relations) with boundary label freely equal to the label of $\partial(\Delta)$ and such that

- no two non-0-edges $e \neq f^{ \pm 1}$ share a vertex,

- no two different non-0-cells share a boundary vertex. 

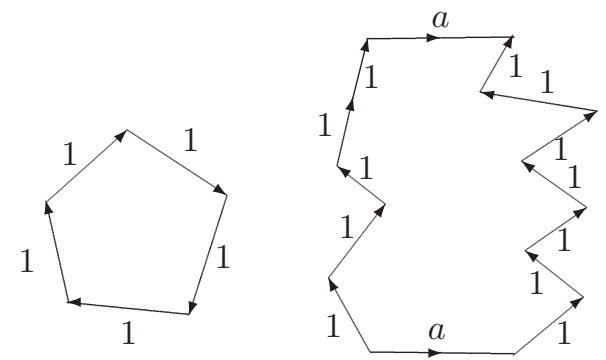

Figure 5. 0-cells.
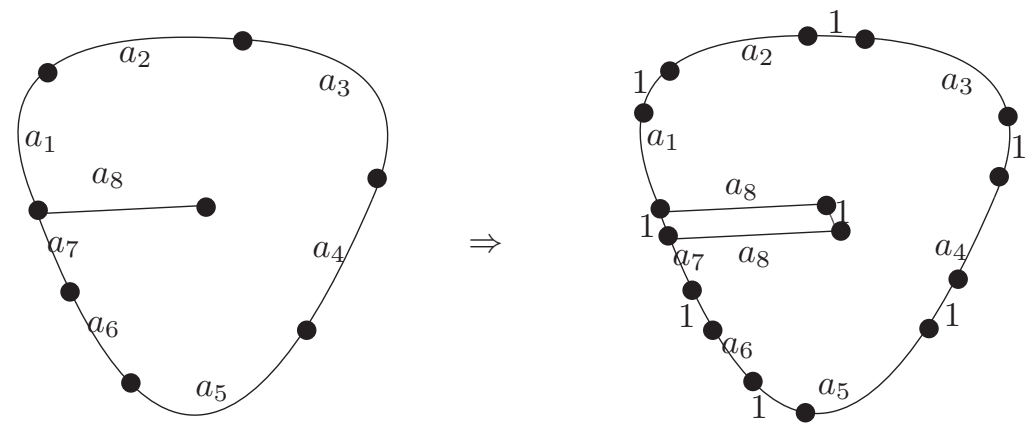

Figure 6. Turning a cell into an embedded disc.
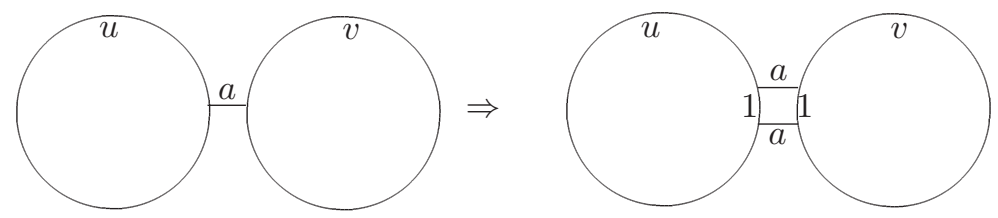

Figure 7. Turning a diagram into an embedded disc.

Making a surgery on a van Kampen diagram, say, replacing a subdiagram with another subdiagram (see below) with the same boundary, we usually first insert 0 -edges and 0-cells, then do the surgery, and then remove 0 -cells and 0-edges. This way we can always deal with embedded discs.

\subsubsection{Combinatorial homotopy of van Kampen diagrams.}

Definition 2.2. A (connected) planar subcomplex without cut-points $\Delta^{\prime}$ of a van Kampen diagram $\Delta$ will be called a holey $M$-subdiagram. If $\Delta$ does not have holes (i.e. is homeomorphic to a disc), it will be called a disc subdiagram or simply a subdiagram. 
Recall that a combinatorial homotopy can insert and delete subdiagrams consisting of two mirror image cells that share an edge and also can make diamond moves [6] as shown on Figure 8 (without 0-cells, diamond moves can be more complicated because the edges involved can be loops, can have two common endpoints, etc.; see [19, p. 17]).
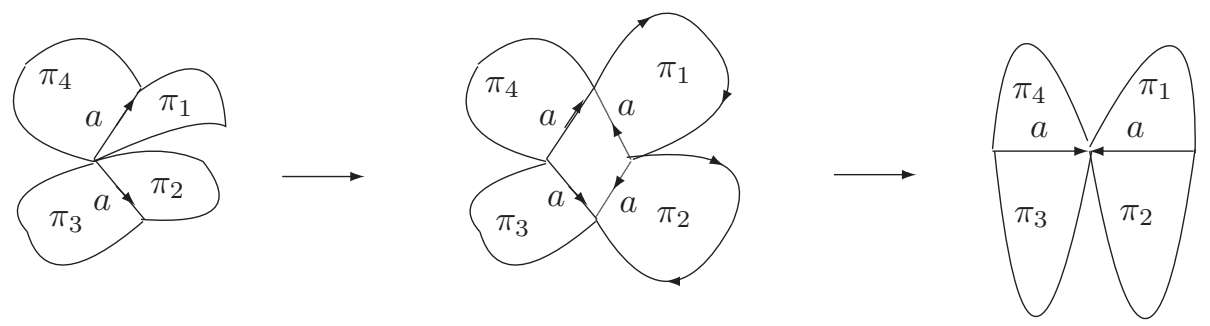

Figure 8. Diamond move: cut along edges labeled by $a$, then fold; $\pi_{1}, \pi_{2}, \pi_{3}, \pi_{4}$ are cells.

A van Kampen diagram is called reduced if it does not have a cancelable pair of cells.

If $\Delta$ is a van Kampen diagram, then $\Delta^{-1}$ is the diagram with the same boundary label as $\Delta$ such that if we glue $\Delta$ and $\Delta^{-1}$ along the boundary, then the resulting van Kampen diagram on a sphere reduces to a diagram without cells.

If $\Gamma$ is an annular diagram obtained by removing a subdiagram $\Delta^{\prime}$ from a van Kampen diagram $\Delta$, then $\Gamma^{-1}$ is the diagram which has the same labels of the outer and inner boundaries and such that if we glue $\Gamma^{-1}$ and $\Gamma$ along the external boundary component and then reduce the resulting diagram, we get a diagram without cells.

2.1.4. The standard surgery on van Kampen diagrams. We shall use the following surgery on van Kampen diagrams. Let $\Delta_{1}, \Delta_{2}$ be van Kampen diagrams over a group presentation $\langle X \mid R\rangle$. Suppose that there exists a subdiagram $\Delta^{\prime}$ of $\Delta$ which is also a subdiagram of $\Delta_{2}$ (see Figure 91). Let $\Gamma$ be the complement of $\Delta^{\prime}$ in $\Delta_{2}$. Then $\Gamma$ is an annular diagram. The standard surgery on $\Delta_{1}$ is the following: cut $\Delta_{1}$ along the boundary of $\Delta^{\prime}$. In the resulting hole insert $\Gamma$ and its inverse $\Gamma^{-1}$ so that if we cancel cells from $\Gamma$ and $\Gamma^{-1}$, we get $\Delta_{1}$ back. The new (non-reduced) diagram is the result of the surgery. Note that it contains $\Delta_{2}$ and $\Gamma^{-1}$ as subdiagrams but has the same boundary label as $\Delta_{1}$ (see Figure 10).

2.1.5. Bands and annuli. The next definition of a band in a diagram is crucial for our paper.

Let $\langle\mathcal{X} \mid R\rangle$ be a group presentation.

Let $S$ be a subset of $\mathcal{X}$. An $S$-band $\mathcal{B}$ is a sequence of cells $\pi_{1}, \ldots, \pi_{n}$ in a van Kampen diagram such that

- each two consecutive cells in this sequence have a common edge labeled by a letter from $S$,

- each cell $\pi_{i}, i=1, \ldots, n$, has exactly two $S$-edges (i.e. edges labeled by a letter from $S$ ) having opposite orientations. 

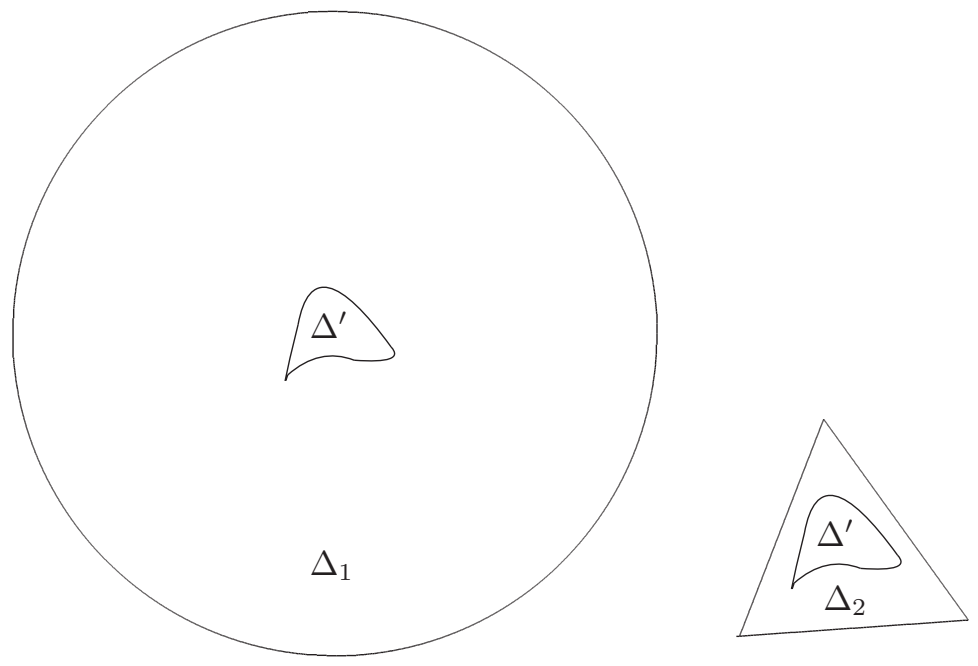

Figure 9. Two van Kampen diagrams with a common subdiagram.

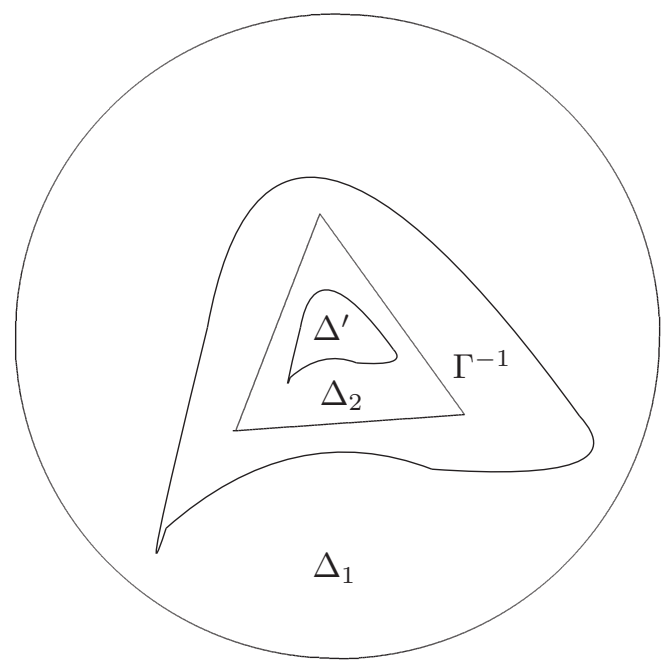

FiguRE 10. The result of a standard surgery. The new diagram has the same boundary label as $\Delta_{1}$ and contains $\Delta_{2}$ inside.

Figure 11 illustrates this concept. In this figure edges $e, e_{1}, \ldots, e_{n-1}, f$ are $S$ edges; the lines $l\left(\pi_{i}, e_{i}\right), l\left(\pi_{i}, e_{i-1}\right)$ connect fixed points in the cells with fixed points of the corresponding edges.

The broken line formed by the lines $l\left(\pi_{i}, e_{i}\right), l\left(\pi_{i}, e_{i-1}\right)$ connecting points inside neighboring cells is called the median of the band $\mathcal{B}$. The $S$-edges $e$ and $f$ are called the start and end edges of the band. The boundary of the subdiagram $\bigcup \pi_{i}$ has the form $e \mathfrak{p} f^{-1} \mathfrak{q}^{-1}$. The paths $\mathfrak{p}, \mathfrak{q}$ are called the sides of the band. 


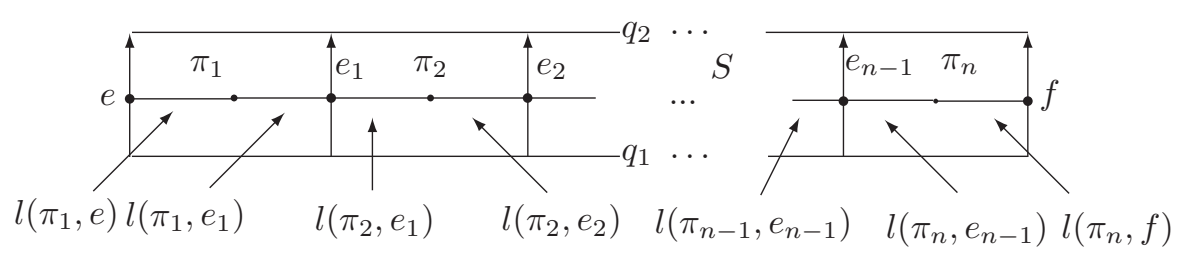

FiguRE 11

A band $\pi_{1}, \ldots, \pi_{t}$ is called reduced if $\pi_{i+1}$ is not a mirror image of $\pi_{i}, i=$ $1, \ldots, t-1$ (otherwise cells $\pi_{i}$ and $\pi_{i+1}$ cancel and there exists a diagram with the same boundary label as $\bigcup_{i} \pi_{i}$ and containing fewer cells).

We say that two bands intersect if their medians intersect. We say that a band is an annulus if its median is a closed curve (see Figure 12(a)).

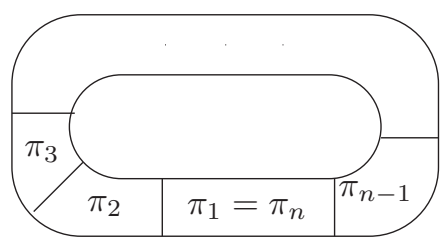

(a)

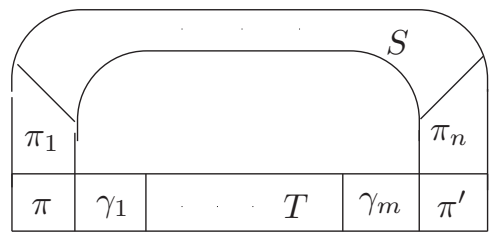

(b)

FiguRE 12

Let $S$ and $T$ be two disjoint sets of letters, let $\left(\pi, \pi_{1}, \ldots, \pi_{n}, \pi^{\prime}\right)$ be an $S$-band, and let $\left(\pi, \gamma_{1}, \ldots, \gamma_{m}, \pi^{\prime}\right)$ be a $T$-band. Suppose that

- the medians of these bands form a simple closed curve,

- on the boundary of $\pi$ and on the boundary of $\pi^{\prime}$ the pairs of $S$-edges separate the pairs of $T$-edges,

- the start and end edges of these bands are not contained in the region bounded by the medians of the bands.

Then we say that these bands form an $(S, T)$-annulus and the closed curve formed by the parts of medians of these bands is the median of this annulus (see the right side of Figure 3). For every annulus we define the inside diagram of the annulus as the subdiagram bounded by the median of the annulus. The union of the inside diagram and the annulus is called the subdiagram bounded by the annulus.

We shall call an $S$-band maximal if it is not contained in any other $S$-band.

2.2. Asphericity and free constructions. Let $\mathcal{P}=\langle X \mid R\rangle$ be a group presentation.

Assumption 2.3. We shall always assume that all words in $R$ are cyclically reduced, no relator is a proper power in the free group, and no relator is a cyclic shift of another relator or its inverse. 
A group presentation $\mathcal{P}$ is called (topologically) aspherical if the universal cover of the presentation complex 3 of $\mathcal{P}$ is contractible. The presentation $\mathcal{P}$ is combinatorially aspherical [6. Section 6] (sometimes also called Peiffer aspherical [4]) if every spherical van Kampen diagram over $\mathcal{P}$ (i.e. a van Kampen diagram on a disc with empty boundary) is (combinatorially) homotopic to a diagram without cells [5], 6].

The following statement was proved in [5] using different terminology (pictures instead of van Kampen diagrams). We are not going to show here that our terminology is equivalent to the one in [5] (but see a translation of that terminology into the language of diagrams in [20, Section 32]). In fact we only need the "only if" implication of that lemma, which is obvious.

Lemma 2.4 ([5, Proposition 1.3]). Under Assumption 2.3 a group presentation is combinatorially aspherical if and only if it is aspherical.

We shall use the following two "combination" statements for asphericity from [5] (see Theorems 3.7 and 4.3 there). Since the terminology used in [5] differs from ours, we provide a proof of the second statement here (the idea of the proof is common for many proofs involving van Kampen diagrams). The proof of the first statement is similar.

Lemma 2.5 (HNN-extensions). Let $\mathcal{P}_{1}=\langle M \mid L\rangle$ be a presentation of a group $T$ and let $\mathcal{P}_{2}=\left\langle M, t \mid L, t x_{i} t^{-1} y_{i}^{-1}, i \in I\right\rangle$ be the standard presentation of an $H N N$ extension of $T$. Suppose that no $t x_{i} t^{-1} y_{i}^{-1}$ is conjugate to any other relator of $\mathcal{P}_{2}$. Then $\mathcal{P}_{2}$ is combinatorially aspherical if $\mathcal{P}_{1}$ is combinatorially aspherical and the images in $T$ of $\left\{x_{i}, i \in I\right\}$ and $\left\{y_{i} \mid i \in I\right\}$ are sets of free generators.

Lemma 2.6 (Amalgamated products). Let $\mathcal{P}=\left\langle M_{1}, M_{2} \mid L_{1}, L_{2}, x_{i} y_{i}^{-1}, i \in I\right\rangle$ be the standard presentation of an amalgamated product where $x_{i} \in M_{1}, y_{i} \in M_{2}$. Then $\mathcal{P}$ is combinatorially aspherical if $\mathcal{P}_{1}=\left\langle M_{1} \mid L_{1}\right\rangle$ and $\mathcal{P}_{2}=\left\langle M_{2} \mid L_{2}\right\rangle$ are combinatorially aspherical, and both $\left\{x_{i}, i \in I\right\}$ and $\left\{y_{i}, i \in I\right\}$ freely generate free subgroups of the groups given by $\mathcal{P}_{1}$ and $\mathcal{P}_{2}$, respectively.

Proof. Let $\Delta$ be a spherical diagram over $\mathcal{P}$. We need to show that $\Delta$ is combinatorially homotopic to a trivial diagram. Recall [18] that $\Delta$ is a labeled graph drawn on a tree of smaller spheres connected by paths. Since it is enough to prove that each of the subgraphs on the smaller spheres is homotopically trivial, we can assume that $\Delta$ is a labeled graph drawn on a sphere and every edge of $\Delta$ belongs to the boundary of a cell.

If $\Delta$ only has cells corresponding to the relations of $\mathcal{P}_{1}$ (of $\mathcal{P}_{2}$ ), then we can use asphericity of $\mathcal{P}_{1}$ (of $\mathcal{P}_{2}$ ). So assume $\Delta$ has cells corresponding to the relations of both $\mathcal{P}_{1}$ and $\mathcal{P}_{2}$. Consider a maximal holey subdiagram $\Delta_{1}$ of $\Delta$ filled with $\mathcal{P}_{1^{-}}$ cells. Since $\Delta_{1} \neq \Delta$, it must have a non-trivial boundary component $\mathfrak{p}$ (possibly more than one) that bounds a subdiagram $\Delta_{2}$ (the one that does not contain $\Delta_{1}$ ) containing cells. Let $u$ be the label of that boundary component. Note that each letter in $u$ belongs to both a relation of $\mathcal{P}_{1}$ and a relation not in $\mathcal{P}_{1}$, which then must be one of the relations $x_{i} y_{i}^{-1}, i \in I$, since $M_{1}, M_{2}$ are disjoint. Hence $u$ is a word in $\left\{x_{i}, i \in I\right\}$. Since $\left\{x_{i}, i \in I\right\}$ freely generates a free subgroup in the group given by $\mathcal{P}_{1}$, it freely generates a free subgroup in the group given by $\mathcal{P}$ (the group given by

\footnotetext{
${ }^{3}$ The presentation complex of $\mathcal{P}$ has one vertex, edges corresponding to the generators of $\mathcal{P}$, and 2-cells corresponding to the relators of $\mathcal{P}$ [18.
} 
$\mathcal{P}_{1}$ embeds into the group given by $\left.\mathcal{P}\right)$. Hence $u$ is freely trivial. Using the diamond moves, one can make the reduced boundary of $\Delta_{2}$ trivial. Since $\Delta_{2}$ has fewer cells than $\Delta$, we can use induction and conclude that $\Delta_{2}$ is combinatorially homotopic to a trivial diagram. Hence $\Delta$ is combinatorially homotopic to a spherical diagram with fewer cells and we can again use induction.

\section{An AUXiLiary Group}

Let $A$ be an alphabet, and let $B, X, Y$ be two-letter disjoint alphabets which are disjoint from $A$. It is easy to find maps $\beta, \beta^{\prime}: A \times X \rightarrow B^{*}, \chi, \chi^{\prime}: A \times X \rightarrow Y^{*}$, $\gamma: B \times Y \rightarrow Y^{*}, \psi: B \times X \rightarrow Y^{*}, \phi: A \times Y \rightarrow B^{*}$ such that the set $\chi(A \times X) \cup$ $\chi^{\prime}(A \times X) \cup \gamma(B \times Y) \cup \psi(B \times X)$ satisfies the small cancelation condition $C^{\prime}\left(\frac{1}{12}\right)$; the set $\beta(A \times X) \cup \beta^{\prime}(A \times X) \cup \phi(A \times Y)$ also satisfies $C^{\prime}\left(\frac{1}{12}\right) 4$

Let $H(A)$ be the group given by the presentation

$$
\begin{aligned}
& \langle A \cup B \cup X \cup Y| x a=\beta(a, x) a \beta^{\prime}(a, x) \chi(a, x) x \chi^{\prime}(a, x), y a=a \phi(a, y) y, \\
& x b=b \psi(b, x) x, y b=b \gamma(b, y), \forall a \in A, x \in X, b \in B, y \in Y\rangle .
\end{aligned}
$$

Figure 13 shows the cells corresponding to the defining relations of $H(A)$. The van Kampen diagrams over the presentation of $H(A)$ are tesselated by these cells.
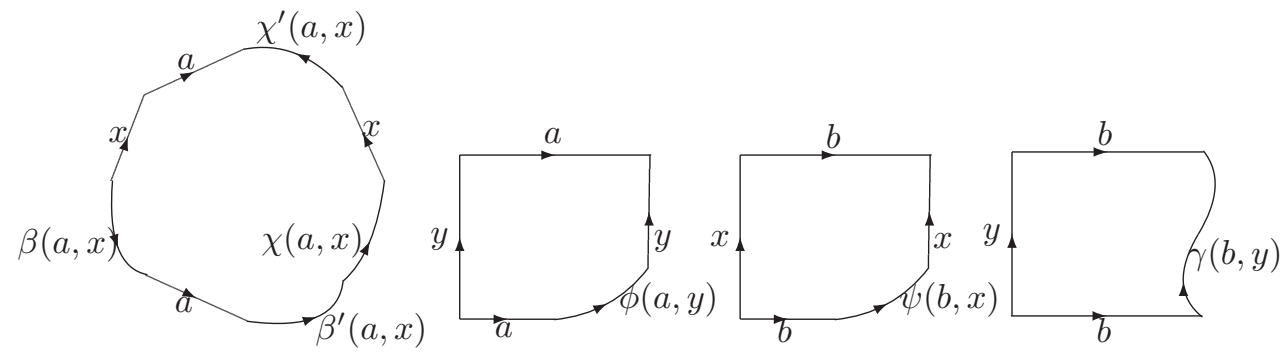

Figure 13. $(A, X)$-cell, $(A, Y)$-cell, $(B, X)$-cell, and $(B, Y)$-cell.

The words $\beta(a, x), \beta^{\prime}(a, x), \chi(a, x), \chi^{\prime}(a, x), \phi(a, y), \psi(a, y), \gamma(b, y)$ are called the large sections of the defining relators and the corresponding sections of boundaries of cells in the van Kampen diagrams will be called large sections of the cells. The following lemma immediately follows from the fact that the set of all large sections of defining relators satisfies the property $C^{\prime}\left(\frac{1}{12}\right)$.

Lemma 3.1. The presentation of $H(A)$ satisfies $C^{\prime}\left(\frac{1}{12}\right)$. If a large section of a cell $\pi$ in a van Kampen diagram $\Delta$ over the presentation of $H(A)$ shares a subpath of at least $\frac{1}{12}$ of its length with the boundary of another cell $\pi^{\prime}$ of $\Delta$, then $\pi, \pi^{\prime}$ cancel.

For every van Kampen diagram $\Delta$ over the presentation of $H(A)$ and every $p \in A \cup B \cup X \cup Y$, we can consider $p$-bands in $\Delta$. We shall also call it an $A$ band ( $B$-band, $X$-band) if $p \in A$ (resp. $B, X)$. The $A$-bands can include $(A, X)$ and $(A, Y)$-cells; $X$-bands can include $(A, X)$ - and $(B, X)$-cells. Maximal $A$ - or $X$-bands can start and end on the boundary of $\Delta$. A $B$-band may contain $(B, X)$-,

\footnotetext{
${ }^{4}$ For every alphabet $T$, the notation $T^{*}$ stands for the set of all words in $T$. A set of words $Q$ satisfies the condition $C^{\prime}(\lambda)$ for some $\lambda>0$ if for every two different cyclic shifts $u, v$ of two words in $Q^{ \pm 1}$, the length of their maximal common prefix is smaller than $\lambda \min \{|u|,|v|\}$.
} 
$(B, Y)$-cells. It can start and end either on $\partial \Delta$ or on the boundary of an $(A, X)$-cell or on the boundary of an $(A, Y)$-cell.

Lemma 3.2. In a reduced 5 van Kampen diagram over $H(A)$, there are no

(1) A-annuli,

(2) X-annuli,

(3) B-annuli,

(4) $(A, X)$-annuli,

(5) $(B, X)$-annuli.

Proof. We prove all five statements by simultaneous induction on the number of cells in the diagram. Suppose that a reduced van Kampen diagram over the presentation of $H(A)$ has an annulus of one of the types mentioned in the lemma. Let $\Delta^{\prime \prime}$ be the inside subdiagram of $\mathcal{A}$, and let $\Delta^{\prime}$ be the diagram bounded by $\mathcal{A}$. We can assume that there is no annulus of one of the types (1)-(5) of the lemma with a smaller inside subdiagram.

(1) Suppose that $\mathcal{A}$ is an $A$-annulus. The diagram $\Delta^{\prime \prime}$ does not contain $(A, X)$ or $(A, Y)$-cells because its boundary does not contain $A$-edges and because, by the minimality assumption, $\Delta^{\prime \prime}$ does not contain $A$-annuli. Hence all cells in $\Delta^{\prime \prime}$ are $(B, X)$ - and $(B, Y)$-cells.

Suppose that $\Delta^{\prime \prime}$ contains cells.

Since $\Delta^{\prime \prime}$ does not contain $B$-annuli, the boundary of $\Delta^{\prime \prime}$ contains $B$-edges. If the diagram $\Delta^{\prime}$ contains an $(A, X)$ - or a $(B, X)$-cell, then the $X$-band containing this cell must intersect $\mathcal{A}$ twice creating an $(A, X)$-annulus with smaller inside subdiagram than $\Delta^{\prime \prime}$. Hence $\Delta^{\prime \prime}$ does not contain $(A, X)$ - or $(B, X)$-cells. Hence all cells in $\Delta^{\prime \prime}$ are $(B, Y)$-cells, and all cells in $\mathcal{A}$ are $(A, Y)$-cells. Since $\Delta^{\prime \prime}$ does not contain $B$-annuli, $\partial \Delta^{\prime \prime}$ must contain $B$-edges, and so the label of $\partial \Delta^{\prime \prime}$ is a product of words from the set $\phi(A \times Y) Y$ and their inverses. By the Greendlinger lemma [18] one of the large sections $\gamma(B \times Y)$ shares a subword of at least $1 / 2$ of its length with the label of $\partial \Delta^{\prime \prime}$, which contradicts the assumption that the set of all large sections satisfies $C^{\prime}\left(\frac{1}{12}\right)$.

If $\Delta^{\prime \prime}$ does not contain cells, then its boundary label is freely trivial and by Lemma 3.1 two neighbor cells in $\mathcal{A}$ cancel.

(2) The case when $\mathcal{A}$ is an $X$-annulus is ruled out similarly.

(3) Suppose that $\mathcal{A}$ is a $B$-annulus. Since $\Delta$ does not have $(B, X)$-annuli with smaller inside subdiagrams than $\Delta^{\prime \prime}, \Delta^{\prime}$ does not contain $(B, X)$-cells. Hence $\Delta^{\prime}$ consists of $(B, Y)$-cells. But then the boundary of $\Delta^{\prime \prime}$ consists of $Y$-edges; hence $\Delta^{\prime \prime}$ does not contain $B$-bands. Thus $\Delta^{\prime \prime}$ does not contain cells. Hence the label of $\partial \Delta^{\prime \prime}$ is freely trivial. The fact that $\gamma(B \times Y)$ satisfies the condition $C^{\prime}\left(\frac{1}{12}\right)$ implies that this set freely generates the free group; hence the label of $\Delta^{\prime}$ is freely trivial as well, and so $\mathcal{A}$ contains a pair of cells that cancel by Lemma 3.1

(4) Suppose that $\mathcal{A}$ is an $(A, X)$-annulus, composed of an $A$-band $\mathcal{A}_{1}$ and a $X$ band $\mathcal{A}_{2}$. By the minimality of $\Delta^{\prime \prime}$, we can assume that $\mathcal{A}$ contains only two cells having $X$ - or $A$-edges, the two common cells $\pi_{1}, \pi_{2}$ of $\mathcal{A}_{1}$ and $\mathcal{A}_{2}$. Hence all cells in $\Delta^{\prime \prime}$ are $(B, Y)$-cells. Now the condition $C^{\prime}\left(\frac{1}{12}\right)$ satisfied by the set of large sections immediately implies that $\Delta^{\prime \prime}$ does not contain cells. Thus the label of the boundary of $\Delta^{\prime \prime}$ is freely trivial. There are four possibilities for the diagram $\Delta^{\prime}$ depending on which edges on $\partial \pi_{1}$ and $\partial \pi_{2}$ are connected by $\mathcal{A}_{1}$ and $\mathcal{A}_{2}$. These possibilities

\footnotetext{
${ }^{5}$ We call a diagram reduced if it does not contain two mirror image cells sharing an edge.
} 


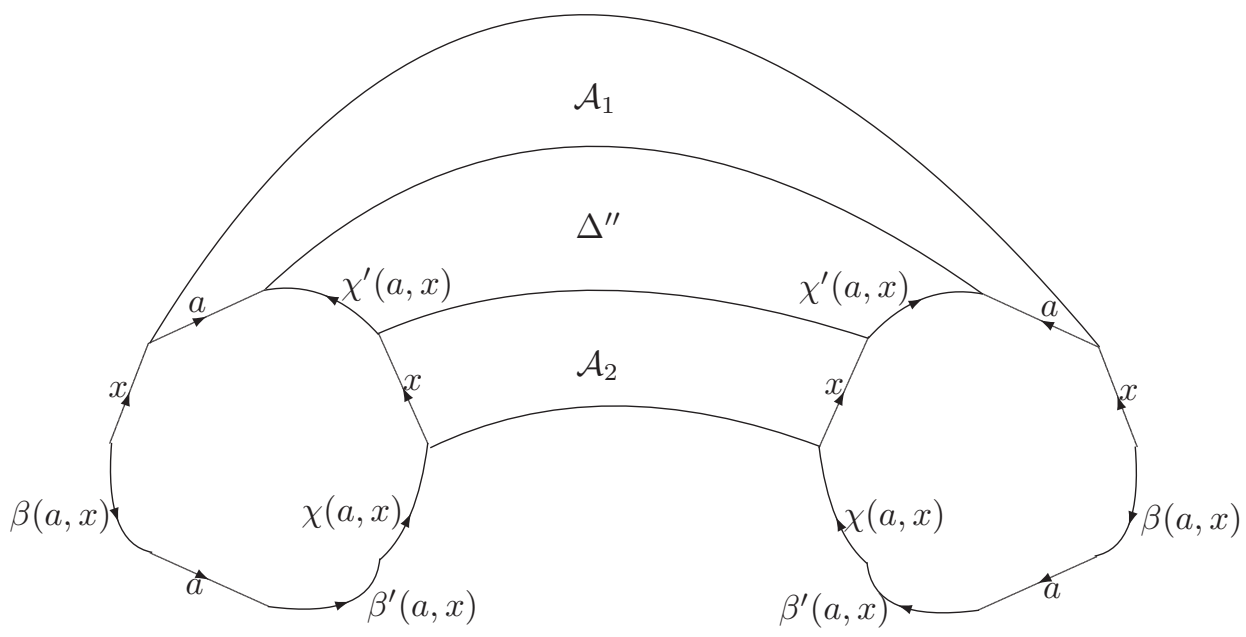

Figure 14

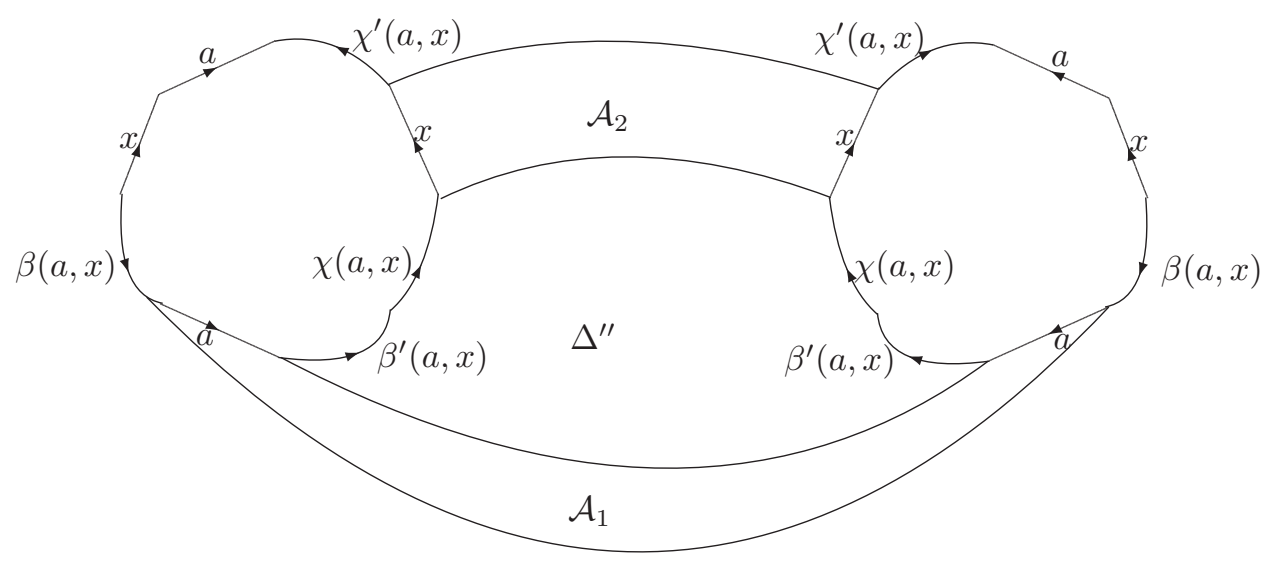

FIGURE 15

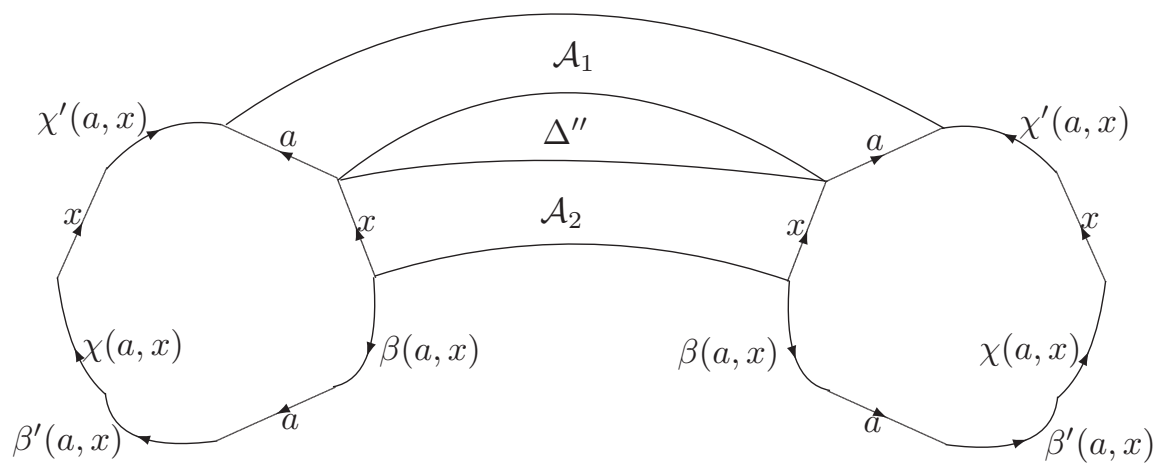

Figure 16 


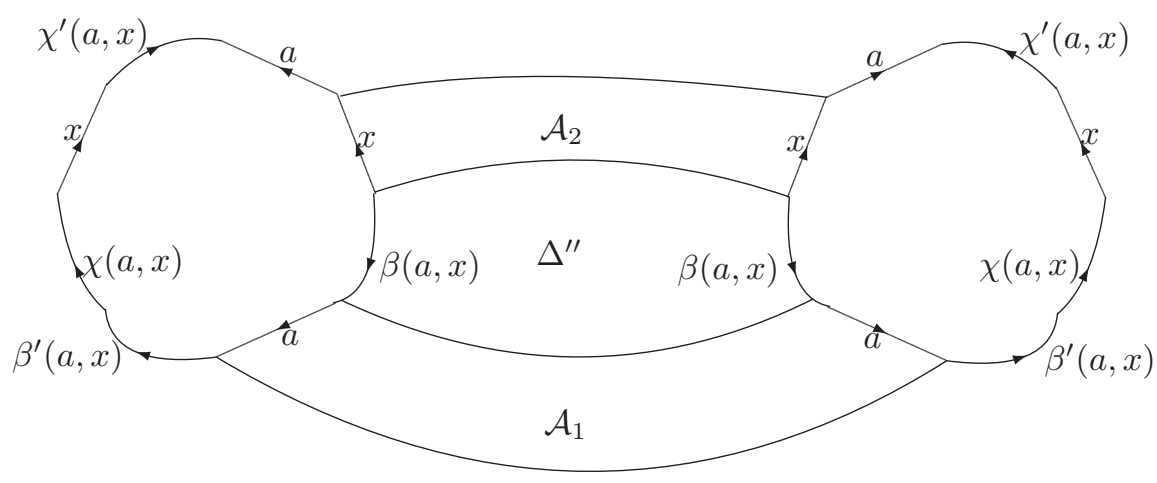

FiguRE 17

are depicted on Figures 14,17] In each of these figures let $\mathfrak{p}_{1}$ be the intersection of $\partial \Delta^{\prime \prime}$ with $\partial \mathcal{A}_{1}$ and let $\mathfrak{p}_{2}$ be the intersection of $\partial \Delta^{\prime \prime}$ with $\mathcal{A}_{2}$. It is easy to see that in each of the four cases $\operatorname{Lab}\left(\mathfrak{p}_{1}\right)$ should be conjugate to $\operatorname{Lab}\left(\mathfrak{p}_{2}\right)$ in the free group. Unless both $\operatorname{Lab}\left(\mathfrak{p}_{1}\right)$ and $\operatorname{Lab}\left(\mathfrak{p}_{2}\right)$ are empty, this leads to a contradiction as follows.

In the case of Figure 14 $\operatorname{Lab}\left(\mathfrak{p}_{1}\right)$ is a product of words from the set $\phi(\{a\} \times Y) Y$ and their inverses, and $\operatorname{Lab}\left(\mathfrak{p}_{2}\right)$ is a word in $B$. In the case of Figure 15, $\operatorname{Lab}\left(\mathfrak{p}_{1}\right)$ is a product of words from the set $\phi(\{a\} \times Y) Y$ and their inverses ( $a$ here is the label of the $a$-edges in $\pi_{1}$ and $\left.\pi_{2}\right)$, and $\operatorname{Lab}\left(\mathfrak{p}_{2}\right)$ is a product of word of the form $B \psi(B \times\{x\})$ and their inverses ( $x$ here is the label of $x$-edges in $\left.\pi_{1}, \pi_{2}\right)$. In the case of Figure 16, $\operatorname{Lab}\left(\mathfrak{p}_{1}\right)$ is a word in $Y$, while $\operatorname{Lab}\left(\mathfrak{p}_{2}\right)$ is a word in $B$. In the case of Figure 17. $\operatorname{Lab}\left(\mathfrak{p}_{1}\right)$ is a word in $Y$, and $\operatorname{Lab}\left(\mathfrak{p}_{2}\right)$ is a product of words from $B \psi(B \times\{x\})$ and their inverses. In each of these cases the words $\operatorname{Lab}\left(\mathfrak{p}_{1}\right)$ and $\operatorname{Lab}\left(\mathfrak{p}_{2}\right)$ are obviously non-conjugate in the free group.

(5) Suppose that $\mathcal{A}$ is a $(B, X)$-annulus composed of a $B$-band $\mathcal{A}_{1}$ and an $X$ band $\mathcal{A}_{2}$. Then $\mathcal{A}_{2}$ cannot contain $(A, X)$-cells (otherwise the $A$-band containing that cell will form an $(A, X)$-annulus with $\mathcal{A}_{2}$ whose inside subdiagram would be smaller than $\left.\Delta^{\prime \prime}\right)$. Hence all cells in $\mathcal{A}_{1}$ are $(B, Y)$-cells and all cells in $\mathcal{A}_{2}$ are ( $B, X)$-cells. If $\mathcal{A}_{2}$ is not empty, then the $B$-band starting on the boundary of a cell in $\mathcal{A}_{2}$ would form a $(B, X)$-annulus with $\mathcal{A}_{2}$ whose inside subdiagram would be smaller than $\Delta^{\prime \prime}$. Thus $\mathcal{A}_{2}$ is empty and $\pi_{1}, \pi_{2}$ cancel, a contradiction.

Lemma 3.3. The sets $A \cup B, X \cup Y$ freely generate free subgroups of $H(A)$, and $\langle A \cup B\rangle \cap\langle X \cup Y\rangle=\{1\}$.

Proof. Suppose that there exists a reduced non-empty diagram $\Delta$ over $H(A)$ with $\partial(\Delta)=\mathfrak{p q}, \operatorname{Lab}(\mathfrak{p})$ is a reduced group word in $A \cup B, \operatorname{Lab}(\mathfrak{q})$ is a reduced group word in $X \cup Y$, and one but not both of these words may be empty. If $\Delta$ contains an $(A, X)$-cell $\pi$, then consider the maximal $A$-band $\mathcal{A}$ containing that cell. That band must start and end on $\mathfrak{p}$ because $q$ does not contain $A$-edges. Then the maximal $X$-band $\mathcal{X}$ containing $\pi$ intersects $\mathcal{A}$. By Lemma 3.2 $\mathcal{X}$ must end on $\mathfrak{p}$. This is a contradiction since $\mathfrak{p}$ does not contain $X$-edges. Hence $\Delta$ does not contain $(A, X)$-cells. 
If $\Delta$ contains an $(A, Y)$-cell $\pi$, then let $\mathcal{A}$ be the maximal $A$-band containing $\pi$. Let $\Delta^{\prime}$ be the subdiagram of $\Delta$ bounded by the median of $\mathcal{A}$ and $\mathfrak{p}$. The boundary of $\Delta^{\prime}$ is of the form $\mathfrak{p}^{\prime} \mathfrak{q}^{\prime}$ where $\mathfrak{p}^{\prime}$ is a subpath of $\mathfrak{p}, \mathfrak{q}^{\prime}$ is a subpath of a side of $\mathcal{A}$. Note that $\mathfrak{q}^{\prime}$ must contain $y$-edges otherwise two neighbor cells in $\mathcal{A}$ cancel (by Lemma 3.10. Since $\mathfrak{p}$ does not contain $y$-edges, the diagram $\Delta^{\prime}$ must contain cells.

We can assume that $\Delta^{\prime}$ does not contain $(A, Y)$-cells (otherwise we can consider a smaller subdiagram). Hence it consists of $(B, Y)$-cells. The condition $C^{\prime}\left(\frac{1}{12}\right)$ implies that the large section of one of the $(B, Y)$-cells in $\Delta$ shares a subpath of at least $\frac{1}{6}$ of its length with $\mathfrak{q}^{\prime}$, which is impossible because the set of all large sections satisfies $C^{\prime}\left(\frac{1}{12}\right)$. This contradiction proves both statements of the lemma.

Definition 3.4. If $W$ is a word in an alphabet $Z, Z^{\prime} \subset Z$, then the projection of $W$ onto the alphabet $Z^{\prime}$ is the word obtained by deleting all letters of $Z \backslash Z^{\prime}$ (and their inverses) from $W$.

Lemma 3.5. Every positive word $W$ in $A \cup B \cup X \cup Y$ is equal in $H(A)$ to a unique word of the form $U V$ where $U \in(A \cup B)^{*}, V \in(X \cup Y)^{*}$. The projections of $U$ and $W$ onto the alphabet $A$ are equal and the projections of $V$ and $W$ on the alphabet $X$ are equal.

Proof. For the proof of existence and the statement about projections, it is enough to prove the following

Claim. For every positive word $W$ in the alphabet $X \cup Y$ and every $p \in A \cup B$ there exist positive words $U \in(A \cup B)^{*}, V \in(X \cup Y)^{*}$ such that

$$
W p=U V
$$

in $H(A)$; the projections onto $X$ of $V$ and $W$ are the same; if $p \in B$, then $U \equiv p$; if $p \in A$, then $U$ contains only one $A$-letter, $p$.

If $W$ is empty, then there is nothing to prove. Let $W \equiv W^{\prime} z$. We consider several cases depending on which set $z$ or $p$ belongs to. In each case, the proof is by induction on the pair $(m, n)$ where $m$ is the number of $X$-letters and $n$ is the number of $Y$-letters in $W$.

Step 1. We prove that the claim is true if $p \in B$. If $m=0$, then the result follows from the $(B, Y)$-relations. Suppose we have proved the statement for $m$ and suppose that $W$ contains $m+1$ occurrences of letters from $X$. Then $W=W^{\prime} x W^{\prime \prime}$ where $x \in X, W^{\prime \prime} \in Y^{*}$. Therefore $W p=W^{\prime} x W^{\prime \prime} p=W^{\prime} x p V_{1}$ for some word $V_{1} \in Y^{*}$ since $W^{\prime \prime}$ does not contain $X$-letters. By the $(p, x)$-relation, we have $W p=W^{\prime} p \psi(p, x) x V_{1}$. Since $W^{\prime}$ has $m X$-letters, the result follows by induction. Thus our claim is true if $p \in B$.

Step 2. We prove that the claim is true if $p \in A, z \in Y$. We have $W p=W^{\prime} z p=$ $W^{\prime} p \phi(p, z) z$. Since $W^{\prime}$ has fewer letters than $W$, we deduce that $W p=U_{1} V_{1} \phi(p, z) z$ where $U_{1} \in(A \cup B)^{*}, V_{1} \in(X \cup Y)^{*}$, the only $A$-letter in $U_{1}$ is $p$, and the number of $X$-letters in $V_{1}$ is the same as in $W^{\prime}$. Since $\phi(p, z) \in B^{*}$, we can finish the proof by applying the statement of Step 1 several times.

Step 3. Finally we prove the claim if $p \in A, z \in X$. In this case we have

$$
W p=W^{\prime} z p=W^{\prime} \beta(p, z) p \beta^{\prime}(p, z) \chi(p, z) z \chi^{\prime}(p, z) .
$$


Since $\beta(p, z)$ is a positive word in the alphabet $B$, we can apply the statement of Step 1 several times, and find $V_{1} \in(X \cup Y)^{*}$ such that $W^{\prime} \beta(p, z)=\beta(p, z) V_{1}$ and the number of $X$-letters in $V_{1}$ is the same as in $W^{\prime}$. Then from (3.1), we obtain

$$
W p=\beta(p, z) V_{1} p \beta^{\prime}(p, z) \chi(p, z) z \chi^{\prime}(p, z) .
$$

Since $V_{1}$ contains fewer $X$-letters than $W$, we can first apply the statements of Steps 2 and 1 and then the inductive hypothesis and obtain that

$$
V_{1} p=U_{1} V_{2}
$$

where $U_{1} \in(A \cup B)^{*}$ contains exactly one $A$-letter, $p$, and $V_{2} \in(X \cup Y)^{*}$ contains the same number of $X$-letters as $V_{1}$. Now from (3.2) we obtain

$$
W p=\beta(p, z) U_{1} V_{2} \beta^{\prime}(p, z) \chi(p, z) z \chi^{\prime}(p, z)
$$

and it remains to apply the statement of Step 1 again several times.

This completes the proof of existence and the statement about projections.

To show uniqueness of $U, V$, we need to prove that there are no reduced group words $U, U^{\prime}$ over $A \cup B$ and $V, V^{\prime}$ over $X \cup Y$ such that $U V=U^{\prime} V^{\prime}$ in $H(A)$ and either $U \neq U^{\prime}$ or $V \neq V^{\prime}$, but that follows immediately from Lemma 3.3 .

Remark 3.6. Since the presentation of the group $H(A)$ satisfies $C^{\prime}\left(\frac{1}{12}\right)$, it is combinatorially aspherical (in fact even diagrammatically aspherical) [18. Hence Lemma 3.5 implies that for every positive $W$ in $A \cup B \cup X \cup Y$ there exists a unique (up to combinatorial homotopy) van Kampen diagram $\Upsilon(W)$ with boundary label $W^{-1} U V$ where $U=U(W)$ is a word in $A \cup B$ and $V=V(W)$ is a word in $X \cup Y$. Words $U, V$ are uniquely determined by $W$.

Remark 3.7. It is easy to see that the lengths of words $U, V$ are in general at least exponential in terms of the length of $W$ (in fact the maximal sum $|U|+|V|$ for $|W|=m$ is at least $\left.\exp O\left(m^{2}\right)\right)$. But the proof of Lemma 3.5 gives an algorithm of computing $U, V$ given $W$.

Notation 3.8. Let $u_{a}, a \in A$, be positive words in $A$ satisfying $C^{\prime}\left(\frac{1}{12}\right)$, and let $v_{a}, a \in A$, be positive words in $X$ satisfying $C^{\prime}\left(\frac{1}{12}\right)$. The words $u_{a}, a \in A$, and $v_{a}, a \in A$, will be called $A$ - and $X$-blocks, respectively. Let $E$ be the subgroup of $H(A)$ generated by $u_{a} v_{a}$. We shall denote $u_{a} v_{a}$ by $\mu(a)$. Let $\mathcal{N}$ be a normal subgroup of $E$ generated by some set $\mu(R)$ where $R$ is a set of words in $A$, and let $\ll \mathcal{N} \gg$ be the normal closure of $\mathcal{N}$ in $H(A)$.

Lemma 3.9. If two words $v_{a}$, $v_{b}$ share a subword of length $\frac{1}{12} \min \left(\left|v_{a}\right|,\left|v_{b}\right|\right)$, then $a=b$ (the same statement for $u$ instead of $v$ is also true).

Proof. Indeed, by $C^{\prime}\left(\frac{1}{12}\right)$ in this case $v_{a} \equiv v_{b}$. Hence $a=b$.

Lemma 3.10. $E$ is a free subgroup of $H(A)$ freely generated by $\{\mu(a), a \in A\}$.

Proof. Indeed, let $\Delta$ be a reduced van Kampen diagram over the presentation of $H(A)$ whose reduced boundary label is a product of words $\mu(a)=u_{a} v_{a}$. If $\Delta$ has cells, then by the Greendlinger lemma [18, one of the cells shares a non-trivial subpath of its large section with $\partial \Delta$, but large sections consist of $B$ - and $Y$-edges, a contradiction. Hence $\Delta$ has no cells and the boundary label of $\Delta$ is freely trivial. The small cancelation condition $C^{\prime}\left(\frac{1}{12}\right)$ then implies that $\partial \Delta$ is empty. Hence the words $\mu(a), a \in A$, freely generate $E$. 
Lemma 3.11. Let $W=\mu(r)$ for some positive word $r$ in the alphabet $A$. Let $U=$ $U(W), V=V(W)$ be the words determined by Lemma 3.5. Then each of the three words $U, V, W$ uniquely determines the two other words and (up to combinatorial homotopy) the diagram over $H(A)$ for the equality $W=U V$.

Proof. Indeed, by Lemma 3.5, the projections of $U$ and $W$ on $A$ coincide. If $r=$ $a_{1} \ldots a_{l}, a_{i} \in A$, then both projections are equal to $u_{a_{1}} \ldots u_{a_{l}}$. Since the words $u_{a_{i}}$ satisfy $C^{\prime}\left(\frac{1}{12}\right), U_{A}$ determines $r$. Hence $U$ determines $W$. Since $W$ determines $V$ by Lemma 3.5, $U$ determines $W$ and $V$. The fact that $V$ determines $U, W$ is proved similarly (one needs to consider the projections onto $X$ ). The fact that $W$ determines $U, V$ is in Lemma 3.5

The fact that $W, U, V$ uniquely (up to combinatorial homotopy) determine the diagram $\Upsilon(W)$ for the equality $W=U V$ is in Remark 3.6 .

Notation 3.12. Consider a van Kampen diagram $\Delta$ over the presentation that consists of the defining relations of $H(A)$ and all reduced words from $\mu(R)$ (Notation 3.8). The cells corresponding to relations from $\mu(R)$ will be called $\mathcal{N}$-cells.

A holey subdiagram of $\Delta$ where the label of each boundary component is freely equal to a product of words $\mu(a)$ and their inverses will be called a holey $E$ subdiagram. In particular, an $\mathcal{N}$-cell is an $E$-subdiagram. Using diamond moves, we can make all boundary components of all maximal holey $E$-subdiagrams reduced. The small cancelation condition $C^{\prime}\left(\frac{1}{12}\right)$ implies that an $A$ - or $X$-block in a product of words of the form $\mu(a)=u_{a} v_{a}$ and their inverses shares less than $1 / 6$ of its length with the neighbor blocks. Thus the reduced label of the boundary component of a holey $E$-subdiagram is a concatenation (without cancelations) of words $u_{a}^{\prime} v_{a}^{\prime}$ and their inverses where $u_{a}^{\prime}$ is a subword of an $A$-block of length at least $5 / 6$ of the length of the block and $v_{a}^{\prime}$ is a subword of an $X$-block of length at least $5 / 6$ of the length of the block. The words $u_{a}^{\prime}, v_{a}^{\prime}$ are called $A$ - and $X$-subblocks. Thus each boundary label of $\Delta^{\prime}$ is a reduced word that is a concatenation of $A$-subblocks and $X$-subblocks.

So we will assume that this property holds and we will call a maximal holey $E$ subdiagrams $\mathcal{N}$-subdiagrams of $\Delta$. Note that by the congruence extension property that we shall prove later, the label of every boundary component of every $\mathcal{N}$ subdiagram is in $\mathcal{N}$ (Lemma 3.15). Consider the graph $\mho=\mho(\Delta)$ whose vertices are the holey $\mathcal{N}$-subdiagrams of $\Delta$. Two vertices are connected if there exists an $X$-band connecting the corresponding subdiagrams. Note that $\mho$ is a planar graph since $X$-bands do not intersect.

We say that two paths $\mathfrak{p}_{1}, \mathfrak{p}_{2}$ in a diagram $\Delta$ share large $X$-portion if $p_{1}=\mathfrak{q}_{1} \mathfrak{p q} q_{1}^{\prime}$, $\mathfrak{p}_{2}=\mathfrak{q}_{2}^{\prime} \mathfrak{p q}_{2}$, and $\operatorname{Lab}(\mathfrak{p})$ is a subword of a word $v_{a}$ (resp. $u_{a}$ ), $a \in A$, of length at least $\frac{1}{12}\left|v_{a}\right|$ (resp. $\left.\frac{1}{12}\left|u_{a}\right|\right)$.

We shall use the following classical result of Heawood [14 several times.

Lemma 3.13. Every non-empty planar graph without loops and multiple edges has a vertex of degree at most 5 .

We shall need the following lemma.

Lemma 3.14. Suppose that $\Delta$ is reduced.

(a) Let $\Pi$ be a holey E-subdiagram of $\Delta$. Then no $X$-band or A-band can start and end on $\partial \Pi$. 
$\left(\mathrm{a}^{\prime}\right)$ Suppose that $\partial \Delta=\mathfrak{p q}$ where $\operatorname{Lab}(\mathfrak{p})$ is a subword of a product of words $\mu(a)$. Then no $X$-band or A-band can start and end on $\mathfrak{p}$.

(b) Let $\Pi, \Pi$ be holey E-subdiagrams in $\Delta$. Let $e_{1}, e_{2}$ be consecutive $X$-edges (resp. A-edges) of $\partial \Pi$ and let the $X$-bands $\mathcal{X}, \mathcal{X}^{\prime}$ starting on $e_{1}$ and $e_{2}$ end on $\partial \Pi^{\prime}$. Then $\mathcal{X}, \mathcal{X}^{\prime}$ are empty bands.

$\left(\mathrm{b}^{\prime}\right)$ Suppose that $\partial \Delta=\mathfrak{p q}$ where $\operatorname{Lab}(\mathfrak{p})$ is a subword of a product of words $\mu(a)$. Let $\Pi$ be a holey E-subdiagram of $\Delta$. Let $e_{1}, e_{2}$ be consecutive $X$-edges (resp. $A$-edges) of $\partial \Pi$ and let the $X$-bands (resp. A-bands) starting on $e_{1}, e_{2}$ end on $\mathfrak{p}$. Then $\mathcal{X}, \mathcal{X}^{\prime}$ are empty bands.

(c) Suppose that $\partial \Delta=\mathfrak{p q}$ where $\operatorname{Lab}(\mathfrak{p})$ is a subword of a product of words $\mu(a)$, $a \in A$, and $\operatorname{Lab}(\mathfrak{q})$ does not have letters from $X$ (resp. letters from A). Suppose $\Delta$ has $\mathcal{N}$-cells. Then there exists a holey $\mathcal{N}$-subdiagram that shares a big $X$-portion (resp. big A-portion) of its boundary with $\mathfrak{p}$.

Proof. We shall prove the five statements by a simultaneous induction on the number of cells in $\Delta$. For diagrams without cells all five statements are obviously true. Suppose that $\Delta$ is a minimal counterexample (with respect to the number of cells).

Suppose that $\Delta$ does not satisfy (a) and that an $X$-band $\mathcal{X}$ starts and ends on the same block subdiagram $\Pi$ in $\Delta$. Let $e_{1}$ be the start edge and let $e_{2}$ be the end edge of $\mathcal{X}$, and (without loss of generality) assume that $e_{1}$ precedes $e_{2}$ in $\partial \Pi$ (traced counterclockwise). Let $\mathfrak{p}$ be the subpath of $\partial \Pi$ between $e_{1}$ and $e_{2}$. Let $\Delta^{\prime}$ be the subdiagram of $\Delta$ bounded by $\mathfrak{p}$ and the median of $\mathcal{X}$. Suppose that $\Delta^{\prime}$ has $\mathcal{N}$-cells. Then by (c) there exists an $\mathcal{N}$-cell $\pi$ that shares a big $X$-portion of its boundary with $\mathfrak{p}$. By Lemma 3.9 the union of $\Pi$ and $\pi$ is an $E$-subdiagram which contradicts maximality of $\Pi$. Hence $\Delta^{\prime}$ does not contain $\mathcal{N}$-cells, so $\Delta^{\prime}$ is a diagram over the presentation of $H(A)$. Therefore, since $X$-bands do not intersect, every $X$-band of $\Delta^{\prime}$ starting on $\mathfrak{p}$ must end on $\mathfrak{p}$. Thus we can assume that there are no $X$-edges on $\mathfrak{p}$.

Hence all edges on $\mathfrak{p}$ (if any) are $A$-edges. Since the boundary of $\Delta^{\prime}$ contains no $X$-edges, $\Delta^{\prime}$ does not contain $(A, X)$ - and $(B, X)$-cells by Lemma 3.2 . Since the presentation of $H(A)$ satisfies $C^{\prime}\left(\frac{1}{12}\right)$ (Lemma 3.1), by the Greendlinger lemma 18 the boundary $\partial \pi_{1}$ of one of the cells in $\Delta^{\prime}$ shares a subpath of length at least $\frac{9}{12}$ of the length of $\partial \pi_{1}$ with the boundary of $\Delta^{\prime}$. Therefore there exists a large section from $\phi(A \times Y)$ or $\gamma(B \times Y)$ which shares a subpath of at least $\frac{1}{6}$ of its length with a product of at most two large sections from the set $\chi^{\prime}(A \times X) \cup \beta(A, X) \cup \beta^{\prime}(A \times$ $X) \cup \chi(A \times X)$. That is impossible because the set of all large sections of defining relators satisfies the small cancelation condition $C^{\prime}\left(\frac{1}{12}\right)$.

The case when $\Delta$ does not satisfy $\left(\mathrm{a}^{\prime}\right)$ is completely analogous to the previous case. Only instead of joining $\Pi$ and $\pi$ together, we would need to cut off $\pi$ from $\Delta$ reducing the number of cells.

Suppose now that $\Delta$ does not satisfy (b). Let $e_{1}, e_{2}$ (resp. $\left.e_{1}^{\prime}, e_{2}^{\prime}\right)$ be the start (resp. end) edges of $\mathcal{X}_{1}, \mathcal{X}_{2}$. We suppose without loss of generality that the labels of $e_{1}, e_{2}$ are positive. We also assume that $e_{1}$ precedes $e_{2}$ on $\partial \Pi$. Then $e_{2}^{\prime}$ precedes $e_{1}^{\prime}$ on $\partial \Pi^{\prime}$. Let $\mathfrak{p}\left(\right.$ resp. $\left.\mathfrak{p}^{\prime}\right)$ be the subpath between $e_{1}, e_{2}$ (resp. $e_{2}^{\prime}$ and $e_{1}^{\prime}$ ) on $\partial \Pi$ (resp. $\partial \Pi^{\prime}$ ). By (c), the subdiagram bounded by the medians of $\mathcal{X}, \mathcal{X}^{\prime}, \mathfrak{p}, \mathfrak{p}^{\prime}$ does not contain $\mathcal{N}$-cells. By (a) $e_{2}^{\prime}, e_{1}^{\prime}$ are consecutive $X$-edges of $\partial \Pi^{\prime}$ (that is, there are no $X$-edges of $\partial \Pi$ between them).

Since $\partial \Delta^{\prime}$ does not contain $X$-edges, $\Delta^{\prime}$ does not contain $(A, X)$ - and $(B, X)$ cells by Lemma 3.2 Thus $\Delta^{\prime}$ is a reduced diagram over the presentation involving 
only $(A, Y)$ - and $(B, Y)$-cells. This and the small cancelation property $C^{\prime}\left(\frac{1}{12}\right)$ imply that $\Delta^{\prime}$ does not have cells. Hence if, say, $\mathcal{X}$ is not empty, a cell in $\mathcal{X}$ shares more than $1 / 12$ of one of its large sections with a large section of another cell of $\mathcal{X}$ or with a large section of a cell in $\mathcal{X}^{\prime}$. By Lemma 3.1, these two cells cancel, a contradiction to the assumption that $\Delta$ is reduced. Hence $\mathcal{X}, \mathcal{X}^{\prime}$ are empty, a contradiction.

Suppose that $\Delta$ does not satisfy $\left(\mathrm{b}^{\prime}\right)$. The only difference with the previous case is that the end edges of $\mathcal{X}$ and $\mathcal{X}^{\prime}$ are consecutive $x$-edges on $\partial \Delta$ in this case, and we need to use $\left(a^{\prime}\right)$ instead of (a). Since the label of $\partial \Delta$ is a product of words of the form $u_{a} v_{a}$ in this case, the proof proceeds the same way as in the previous case.

Finally suppose that $\Delta$ does not satisfy $(\mathrm{c})$. Assume Lab(q) does not have letters from $X$ (the other case is similar).

By (a), the graph $\mho$ from Notation 3.12 does not have a vertex connected to itself. By Lemma 3.13, $\mho$ has a vertex of degree at most 5 .

Suppose that $\Delta$ contains two $\mathcal{N}$-subdiagrams $\Pi$ and $\Pi^{\prime}$ such that at least one fifth of all $X$-bands starting on $\partial \Pi$ end on $\partial \Pi^{\prime}$. By (b), then $\Pi$ and $\Pi^{\prime}$ share large $X$-portions of their boundaries. Therefore the boundaries of the union of $\Pi$ and $\Pi^{\prime}$ have labels from $E$ (by Lemma 3.9), which contradicts the maximality of $\Pi$ and $\Pi^{\prime}$ (as vertices of $\mho$ ).

Therefore there is an $\mathcal{N}$-cell in $\Delta$ and at least one fifth of all $X$-bands starting on $\partial \Pi$ end on $\partial \Delta$. Hence there exists a subpath $\mathfrak{v}$ on the boundary whose label is an $X$-subblock $\mathfrak{w}$ and at least $|\mathfrak{w}| / 5$ of the consecutive $X$-bands starting on $\mathfrak{w}$ end on $\partial \Delta$. By $\left(a^{\prime}\right)$, the end edges of these bands are consecutive edges of $\partial \Delta$. Therefore the end edges of these bands form a path $\mathfrak{p}$ whose label is Lab( $\mathfrak{w})$. By $\left(b^{\prime}\right)$ all these $X$-bands are empty, so $\Pi$ shares a big portion of its boundary with $\mathfrak{p}$, a contradiction.

Lemma 3.15. The subgroup $E$ of $H(A)$ satisfies the congruence extension property; that is, the intersection of $\ll \mathcal{N} \gg$ with $E$ is $\mathcal{N}$ (i.e. $E / \mathcal{N}$ naturally embeds into $H(A) / \ll \mathcal{N} \gg$ ) for every normal subgroup $\mathcal{N}$ of $E$.

Proof. Suppose that $W \in E$ belongs to $\langle\mathcal{N} \gg$. Then we may assume that $W$ is a product of words $u_{a} v_{a}$ and their inverses and that there exists a van Kampen diagram $\Delta$ over the presentation that consists of the defining relations of $H(A)$ and all words from $\mathcal{N}$ and boundary label $W$. We need to show that $W \in \mathcal{N}$. By contradiction assume that $W$ is a counterexample and $\Delta$ is a minimal (with respect to the number of cells) diagram for $W=1$ for all counterexamples $W$. If $\Delta$ does not contain $\mathcal{N}$-cells, then $\Delta$ is a diagram over the presentation of $H(A)$ and so it does not contain cells by Lemma 3.10 .

Suppose that $\Delta$ contains $\mathcal{N}$-cells. Consider the graph $\mho$ as in Notation 3.12. By Lemma 3.13 there exists a vertex $\Pi$ in $\mho$ of degree at most 5 . Therefore at least $\frac{1}{5}$ of the consecutive $X$-bands starting on $\partial \Pi$ end either on the boundary of another $\mathcal{N}$-subdiagram $\Pi^{\prime}$ or on $\partial \Delta$. The first possibility would mean, by Lemma 3.14 . that $\Pi$ and $\Pi^{\prime}$ share large $X$-portions of their boundaries. Hence by Lemma 3.9 the union of $\Pi$ and $\Pi^{\prime}$ is a holey $E$-subdiagram of $\Delta$ which contradicts the maximality of $\Pi, \Pi^{\prime}$. If the second possibility occurs, then by Lemma $3.14\left(b^{\prime}\right), \Pi$ shares a large $X$-portion of its boundary with the boundary of $\Delta$. Then (again by Lemma 3.9) we can cut $\pi$ off $\Delta$ and produce a smaller diagram $\Delta^{\prime}$ with boundary label in $E$. By the minimality assumption for $\Delta, \operatorname{Lab}\left(\partial \Delta^{\prime}\right)$ must belong to $\mathcal{N}$. But then $W \in \mathcal{N}$ as well, a contradiction. 
Lemma 3.16. For every normal subgroup $\mathcal{N}$ of $E,\langle A \cup B\rangle \cap \ll \mathcal{N} \gg=\{1\}$, $\langle X \cup Y\rangle \cap \ll \mathcal{N} \gg=\{1\}$.

Proof. Suppose that the boundary label of $\Delta$ is a reduced group word in $A \cup B$. By Lemma 3.14 (c), $\Delta$ has no $\mathcal{N}$-cells. If $\Delta$ has $(A, X)$-cells or $(B, X)$-cells, then it has $X$-bands, which must start and end on the boundary of $\Delta$ by Lemma 3.2, a contradiction. Hence $\Delta$ consists of $(B, Y)$-cells. These relations satisfy the small cancelation condition $C^{\prime}\left(\frac{1}{12}\right)$. By the Greendlinger lemma [18] there exists a cell $\pi$ in $\Delta$ such that at least $\frac{9}{12}$ of $\partial \pi$ is contained in $\partial \Delta$. But $\frac{9}{12}$ of the boundary of a $(B, Y)$-cell contains $Y$-edges, a contradiction.

Suppose that the boundary label of $\Delta$ is a reduced word in $X \cup Y$. As in the previous paragraph, $\Delta$ does not contain $\mathcal{N}$-cells, $(A, X)$-cells, and $(A, Y)$-cells. Therefore $\Delta$ contains only $(B, X)$ - and $(B, Y)$-cells. Then the $B$-bands must start and end on the boundary of $\Delta$ by Lemma 3.2, a contradiction.

Lemma 3.17. For every word $U$ in $\langle A \cup B\rangle$ there exists at most one pair of reduced words $W \in E$ and $V \in\langle X \cup Y\rangle$ such that $W=U V$ in $H(A)$.

Proof. Indeed, if $W=U V$ and $W^{\prime}=U V^{\prime}$, then $W^{-1} W^{\prime}=V^{-1} V^{\prime} \in\langle X \cup Y\rangle \cap E=$ $\{1\}$ by Lemma 3.16 (take $\mathcal{N}=E$ there). Hence $W=W^{\prime}, V=V^{\prime}$ in the free group.

Notation 3.18. Consider any group $\Gamma=\langle A \mid R\rangle$. We always assume that the presentation of $\Gamma$ is positive, i.e. consists of positive words in the alphabet $A$. For this, we assume that $A$ is divided into two parts of equal sizes $A^{+}, A^{-}$with a bijection $^{-}: A^{+} \leftrightarrow A^{-}$and that $R$ contains all relations of the form $a \bar{a}=1, a \in A$. Clearly every group with combinatorially aspherical presentation has a positive combinatorially aspherical presentation.

Let $A^{\prime}$ be a copy of $A$, let $\varepsilon$ be a bijection $A \rightarrow A^{\prime}$, and let $R^{\prime}=\varepsilon(R)$ be the set of words $R$ rewritten in $A^{\prime}, \Gamma^{\prime}=\left\langle A^{\prime} \mid R^{\prime}\right\rangle$.

Let $H^{\prime}(\Gamma)$ be the group $\left\langle\Gamma^{\prime}, H(A), q \mid q \varepsilon(a) q^{-1}=\mu(a), a \in A^{\prime}\right\rangle$; that is, $H^{\prime}(\Gamma)$ is the factor-group of the free product $\Gamma^{\prime} * H(A) * \mathbb{Z}$ (where the copy of $\mathbb{Z}$ is generated by $q$ ) by the conjugacy $q$-relations $q \varepsilon(a) q^{-1}=\mu(a)$.

Lemma 3.19. Let $\mathcal{N}$ be the normal subgroup of $H(A)$ generated (as a normal subgroup) by $\mu(R), H^{\prime}=H(A) / \mathcal{N}$. The group $H^{\prime}(\Gamma)$ is isomorphic to the $H N N$ extension of the free product $H^{\prime} * \Gamma$ with free letter $q$ and associated subgroups $\Gamma^{\prime}$ and $\langle\mu(A)\rangle \mathcal{N} / \mathcal{N}$.

Proof. Indeed it is enough to establish that the groups $\Gamma$ and $\langle\mu(A)\rangle \mathcal{N} / \mathcal{N}$ are isomorphic. But that follows from the congruence extension property satisfied by the subgroup $E=\langle\mu(A)\rangle$ in $H(A)$ by Lemma 3.15 .

Relations of $H^{\prime}(\Gamma)$ and cells in the van Kampen diagram corresponding to the relations from $R$ of $\Gamma$ will be called $\Gamma$-relations and $\Gamma$-cells.

Lemma 3.20. Suppose that every disc subdiagram $\Delta^{\prime}$ of a diagram $\Delta$ whose boundary consists of $A^{\prime}$-edges consists of $\Gamma$-cells. Suppose also that $\partial \Delta$ does not consist of $A^{\prime}$-edges and does not contain q-edges. Then every $\Gamma$-cell in $\Delta$ is in the inside diagram of a q-annulus of $\Delta$. 
Proof. Indeed, let $\Delta_{1}$ be a maximal holey subdiagram of $\Delta^{\prime}$ that consists of $\Gamma$-cells. Every boundary component of $\Delta_{1}$ consists of $A^{\prime}$-edges. By our assumption, $\Delta_{1}$ does not have holes and is a disc subdiagram. At least one edge on $\partial \Delta_{1}$ must belong to the boundary of a $q$-cell (since $\partial \Delta^{\prime}$ does not consist of $A^{\prime}$-edges). The $q$-band containing that cell is an annulus since $\partial \Delta$ does not contain $q$-edges. That annulus cannot have an outside boundary consisting of $A^{\prime}$-edges by our assumption (see Figure 18(a)). So its inside diagram must contain $\Delta_{1}$ (see Figure 18(b)).

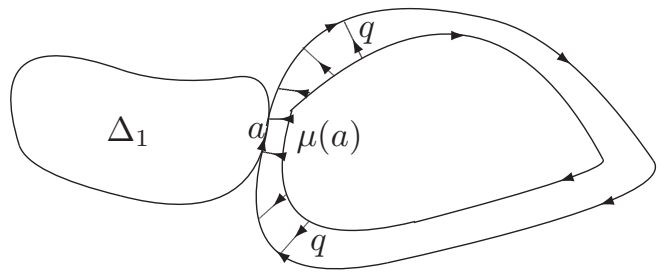

(a)

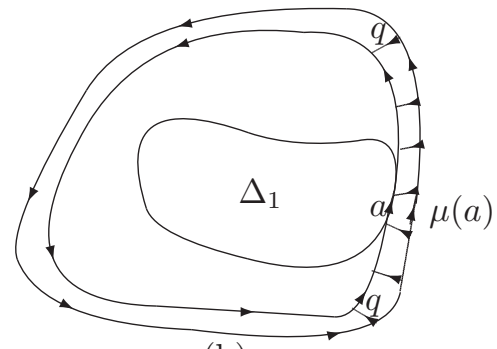

(b)

FIGURE 18

Lemma 3.21. Every diagram over the presentation of $H^{\prime}(\Gamma)$ whose boundary consists of $A^{\prime}$-edges is combinatorially homotopic to a diagram consisting of $\Gamma$-cells. The subset $X \cup Y$ of $H^{\prime}(\Gamma)$ freely generates a free subgroup.

Proof. Let $H^{\prime \prime}$ be the group given by the presentation of $H^{\prime}(\Gamma)$ without the $\Gamma$ relations. Then $H^{\prime \prime}$ is the HNN-extension of the free product $\left\langle A^{\prime}\right\rangle * H(A)$ with free letter $q$ and associated subgroups $\left\langle\varepsilon(A)^{(1)}\right\rangle$ and $\left\langle\mu(A)^{(1)}\right\rangle$ which are both free and freely generated by their respective generating sets by the definition of $\varepsilon$ and $\mu$. Hence by Lemmas 2.5 and [2.6. the presentation of $H^{\prime \prime}$ is aspherical. The standard properties of HNN-extensions and free products show that the set $A^{\prime}$ freely generates a free subgroup in $H^{\prime \prime}$.

With every diagram $\Delta$ over the presentation of $H^{\prime}(\Gamma)$ we associate its 2-weight, a pair of numbers $(m, n)$ where $n$ is the number of $\Gamma$-cells in $\Delta$ and $m$ is the number of other cells in $\Delta$. We order all pairs $(m, n)$ lexicographically.

Now consider any reduced van Kampen diagram $\Delta$ over the presentation of $H^{\prime}(\Gamma)$ whose boundary consists of $A^{\prime}$-edges. Suppose that $\Delta$ is not combinatorially homotopic to a diagram containing only $\Gamma$-cells and that it is a smallest 2 -weight diagram with this property and boundary label in $A^{\prime}$.

If $\Delta$ does not contain $\Gamma$-cells, then the diagram is combinatorially homotopic to the trivial diagram because it is a diagram over the presentation of $H^{\prime \prime}$.

Suppose that $\Delta$ contains $\Gamma$-cells.

If $\Delta$ does not contain conjugacy $q$-cells, then the boundary of every maximal holey subdiagrams of $\Delta$ consisting of $\Gamma$-cells must coincide with the boundary of $\Delta$; therefore $\Delta$ consists of $\Gamma$-cells. Thus we can assume that $\Delta$ contains conjugacy $q$-cells. Hence $\Delta$ has a $q$-annulus $\mathcal{A}$ consisting of conjugacy $q$-cells. Then the label of the outer boundary of $\mathcal{A}$ is either a word in $A^{\prime}$ or is from $E$. Suppose that the first option holds. If $\partial \Delta$ is not the outer boundary of $\mathcal{A}$, we can use the minimality of $\Delta$ and conclude that the diagram bounded by the outer boundary of $\mathcal{A}$ is combinatorially homotopic to a diagram consisting of $\Gamma$-cells. That would 
reduce the number of non- $\mathcal{N}$-cells in $\Delta$ and the 2-weight of $\Delta$, which is impossible. Thus in the first case $\partial \Delta$ is the outer boundary of $\mathcal{A}$. The boundary label of the inside subdiagram $\Delta^{\prime}$ bounded by the median of $\mathcal{A}$ is from $E$. If the second option is true, we can take $\Delta^{\prime}$ to be the subdiagram bounded by the outer boundary of $\mathcal{A}$. Note that in both cases $\Delta^{\prime}$ contains cells.

If $\Delta^{\prime}$ does not contain conjugacy $q$-cells, then it cannot contain $\Gamma$-cells either (otherwise consider a maximal holey subdiagram consisting of $\Gamma$-cells), and so it is a diagram over the presentation of $H(A)$. Since the group $E=\langle\mu(A)\rangle$ in $H(A)$ is free (by Lemma 3.10), we would have that the label of $\Delta^{\prime}$ is freely trivial, so $\mathcal{A}$ contains two neighbor cells that cancel. Hence we can assume that $\Delta^{\prime}$ contains conjugacy $q$-cells and $q$-annuli. By the minimality of $\Delta$, the external boundary of each of these annuli must have label from $E$ and the internal boundary must consist of $A^{\prime}$-edges. By Lemma 3.19 the label of the external boundary of any $q$-annulus in $\Delta$ must then be a word from $\mathcal{N}$.

By Lemma 3.20, every $\Gamma$-cell in $\Delta^{\prime}$ is in the inside subdiagram of a $q$-annulus. Maximal subdiagrams of $\Delta^{\prime}$ bounded by $q$-annuli will be called $q$-subdiagrams; the $q$-annulus bounding a $q$-subdiagram is called the main $q$-annulus of the subdiagram. Using diamond moves, we can make the boundary of every $q$-subdiagram reduced. Note that all $q$-subdiagrams are $E$-subdiagrams of $\Delta$ as in Notation 3.12

The diagram $\Delta^{\prime}$ is tesselated by $q$-subdiagrams and cells corresponding to the relations of $H(A)$, so the situation is the same as the one considered in Notation 3.12 since we can view $\Delta^{\prime}$ as a reduced diagram over the presentation consisting of $\mathcal{N}$-relations and relations from $H(A)$.

As in Notation 3.12 consider the graph $\mho$ whose vertices are all $q$-subdiagrams of $\Delta^{\prime}$ bounded by $q$-annuli and for which two vertices are connected if there is an $X$-band connecting them. Since $\mho$ is a planar graph without multiple edges and vertices adjacent to themselves (by Lemma 3.14(a)), there must be (by Lemma 3.13) a vertex of degree at most 5 . Hence either at least $\frac{3}{12}$ of the consecutive $X$-bands starting on one of the $X$-blocks of the boundary of a $q$-subdiagram $\Pi_{1}$ end on an $X$-block of the boundary of another $q$-subdiagram $\Pi_{2}$ or $\frac{3}{12}$ of the $X$-bands starting on one of the $X$-blocks of the boundary of a $q$-subdiagram $\Pi_{1}$ end on one of the $X$-blocks of the boundary of $\Delta^{\prime}$. By Lemma 3.14(b) the $X$-bands connecting these blocks are empty. Therefore $\Pi_{1}$ shares a large $X$-portion of its boundary either with another $q$-subdiagram $\Pi_{2}$ or with the boundary of $\Delta^{\prime}$. Applying Lemma 3.9. we conclude that in the first case a cell of the main $q$-band of $\Pi_{1}$ cancels with a cell of the main $q$-band of $\Pi_{2}$, and, in the second case, a cell of the main $q$-band of $\Pi_{1}$ cancels with a cell in $\mathcal{A}$. This contradiction completes the proof of the first statement of the lemma.

The second statement immediately follows from Lemmas 3.19 and 3.16 .

\section{The MAIN CONSTRUCTION}

Let $\Gamma=\langle A \mid R\rangle$ be a finitely generated recursively presented group, so that $R$ is a recursive set of defining relations. In this section, we describe an embedding of $\Gamma$ into a finitely presented group $\mathcal{G}$.

\subsection{Preliminaries on $S$-machines.}

4.1.1. A definition of $S$-machines. Following [29, 2] we shall give two (equivalent) definitions of $S$-machines (a slightly different definition can be found in [24, 23, 
[26] and other papers, but since we are going to use the results of [29], we give definitions that are closer to 29]). Let $n$ be a natural number. A hardware of an $S$-machine is a pair $(Z, Q)$ where $Z$ is an $(m-1)$-vector of (not necessary disjoint) sets $Z_{i}$ of tape letters and $Q$ is an $m$-vector of disjoint sets $Q_{i}$ of state letters. The sets $\bigcup Q_{i}$ and $\bigcup Z_{i}$ are also disjoint.

The language of admissible words is $L(\mathcal{S})=Q_{1} F\left(Z_{1}\right) Q_{2} \ldots F\left(Z_{n-1}\right) Q_{m}$ where $F\left(Z_{j}\right)$ is the language of all reduced group words in the alphabet $Z_{j} \cup Z_{j}^{-1}$.

If $1 \leq i \leq j \leq m$ and $W=q_{1} u_{1} q_{2} \ldots u_{m-1} q_{m}$ is an admissible word, $q_{i} \in Q_{i}, u_{i} \in$ $\left(Z_{i} \cup Z_{i}^{-1}\right)^{*}$, then the subword $q_{i} u_{i} \ldots q_{j}$ of $W$ is called the $\left(Q_{i}, Q_{j}\right)$-subword of $W$.

An $S$-machine with hardware $\mathcal{S}$ is a rewriting system. The objects of this rewriting system are all admissible words.

The rewriting rules, or $S$-rules, have the following form:

$$
\left[U_{1} \rightarrow V_{1}, \ldots, U_{n} \rightarrow V_{n}\right]
$$

where the following conditions hold:

- Each $U_{i}$ is a subword of an admissible word starting with a $Q_{\ell^{-}}$-letter and ending with a $Q_{r}$-letter $\ell=\ell(i), r=r(i)$.

- If $i<j$, then $r(i)<\ell(j)$.

- Each $V_{i}$ is also a subword of an admissible word whose $Q$-letters belong to $Q_{\ell(i)} \cup \ldots \cup Q_{r(i)}$ and which contains a $Q_{\ell(i)}$-letter and a $Q_{r(i)}$-letter.

- $V_{1}$ must start with a $Q_{1}$-letter and $V_{n}$ must end with a $Q_{m}$-letter.

- If some $Q_{i}$ does not have a representative that appears in any $U_{j}$, then we assume that the rule includes $q \rightarrow q$ for every $q \in Q_{i}$.

To apply an $S$-rule to a word $W$ means to replace simultaneously subwords $U_{i}$ by subwords $V_{i}, i=1, \ldots, n$. In particular, this means that our rule is not applicable if one of the $U_{i}$ 's is not a subword of $W$. The following convention is important:

After every application of a rewriting rule, the word is automatically reduced.

With every $S$-rule $\tau$ we associate the inverse $S$-rule $\tau^{-1}$ in the following way: if

$$
\tau=\left[U_{1} \rightarrow x_{1} V_{i}^{\prime} y_{1}, U_{2} \rightarrow x_{2} V_{2}^{\prime} y_{2}, \ldots, U_{n} \rightarrow x_{n} V_{n}^{\prime} y_{n}\right]
$$

where $V_{i}^{\prime}$ starts with a $Q_{\ell(i)}$-letter and ends with a $Q_{r(i)}$-letter, then

$$
\tau^{-1}=\left[V_{1}^{\prime} \rightarrow x_{1}^{-1} U_{1} y_{1}^{-1}, V_{2}^{\prime} \rightarrow x_{2}^{-1} U_{2} y_{2}^{-1}, \ldots, V_{n}^{\prime} \rightarrow x_{n}^{-1} U_{n} y_{n}^{-1}\right] .
$$

It is clear that $\tau^{-1}$ is an $S$-rule, $\left(\tau^{-1}\right)^{-1}=\tau$, and that rules $\tau$ and $\tau^{-1}$ cancel each other (meaning that if we apply $\tau$ and then $\tau^{-1}$, we return to the original word).

The following convention is also important:

We always assume that an $S$-machine is symmetric, that is, if an $S$ machine contains a rewriting rule $\tau$, it also contains the rule $\tau^{-1}$. Among any pair of mutually inverse rules we pick one which we call the positive rule; the other rule is called negative.

We define the history of a computation of an $S$-machine as the sequence (word) of rules used in this computation. A computation is called reduced if the history of this computation is reduced, that is, if two mutually inverse rules are never applied next to each other.

For some $S$-machines we distinguish input and stop admissible words. Properties of these words are listed below. 
Remark 4.1. We always assume that:

- There is only one stop word; it does not contain tape letters.

- If we remove tape letters from any two input words, we obtain the same word $q_{1} \ldots q_{m}$ which we call the input base.

- A negative rule cannot apply to any input word.

- There exists only one positive rule that applies to an input word. It has the form $\left[q_{1} \rightarrow \tilde{q}_{1}, \ldots, q_{m} \rightarrow \tilde{q}_{m}\right]$ where $\tilde{q}_{i} \neq q_{i}$ for some $i$ (it just replaces the state letters of the input word by different state letters). This rule will be called the transition rule of the $S$-machine.

If an $S$-machine $\mathcal{S}$ has a stop word $W_{0}$, then we say that an admissible word $W$ is accepted by $\mathcal{S}$ if there exists a computation of $\mathcal{S}$ starting with $W$ and ending with $W_{0}$. That computation is called an accepting computation for $W$.

4.1.2. Recursively enumerable sets and $S$-machines. One of the main results of [29] implies that for every recursively enumerable set of words $L$ in an alphabet $A$ there exists an $S$-machine $\mathcal{S}$ recognizing $L$ in the following sense 6

Proposition 4.2. The $S$-machine $\mathcal{S}$ has a stop word $W_{0}$. For every positive word $u$ in the alphabet $A$, there exists an input word $I(u)$ and the following hold:

(1) The input word $I(u)$ has the form $q_{1} u q_{2} \ldots q_{m}, q_{i} \in Q_{i}, i=1, \ldots, m$.

(2) A word $u$ belongs to $L$ if and only if $I(u)$ is accepted by $\mathcal{S}$.

(3) If $I(u)$ is accepted by $\mathcal{S}$, then there exists only one reduced computation accepting $I(u)$.

(4) There is only one reduced computation connecting $W_{0}$ with itself, the empty one.

Proof. Take a deterministic Turing machine $T$ recognizing $L$. Convert it into a symmetric Turing machine $T^{\prime}$ using [29, Lemma 3.1]. Then use [29, Proposition 4.1] to convert $T^{\prime}$ into an $S$-machine 7 The fact that this $S$-machine satisfies conditions (1)-(4) of the proposition immediately follows from [29, Lemma 3.1 and Proposition 4.1].

4.1.3. S-machines as HNN-extensions of free groups. Another, probably even easier, way to look at $S$-machines is to consider them as multiple HNN-extensions of free groups (see [26, 27]). Let $\mathcal{S}$ be an $S$-machine with the set of tape letters $Z=\bigcup_{i=1}^{m-1} Z_{i}$, the set of state letter $8=\bigsqcup_{i=1}^{m} Q_{i}$, and the set of rules $\Theta$. The set of all positive rules is denoted by $\Theta^{+}$.

The generating set of the group is $Q \cup Z \cup \Theta^{+}$. The relations are

$$
U_{i} \theta=\theta V_{i}, \quad i=1, \ldots, n
$$

\footnotetext{
${ }^{6}$ More precisely, the $S$-machine $\mathcal{S}$ we use is the $S$-machine from 29 without $\alpha$ - and $\omega$-sectors. These sectors are needed in [29] only to control the Dehn function of the resulting group. The facts from 29] that we are using here remain true. We could use literally the same $S$-machines as in 29 but it would make our proof unnecessarily more cumbersome because the input words would contain powers of $\alpha$ and $\omega$.

${ }^{7}$ The conversion in 29] was very complicated because we needed to control the speed of the $S$-machine. Since we do not care about the speed of the $S$-machines in this paper, we could use the simpler but (exponentially) slower $S$-machines constructed in [24]. Still the $S$-machine from 29 is useful for us because we can use some facts about it proved in [29].

${ }^{8} \sqcup$ denotes the disjoint union.
} 
(these relations will be called $(Q, \Theta)$-relations) and

$$
\theta a=a \theta
$$

for all $a \in Z, \theta \in \Theta^{+}$(these relations will be called $(Z, \Theta)$-relations).

For simplicity and following [26], 27], we shall call this group an $S$-machine too and denote it by the same letter $\mathcal{S}$.

Lemma 4.3 ([29, Lemma 7.6]). In any reduced diagram over the presentation of an $S$-machine $\mathcal{S}$, there are no $\Theta$-annuli, $Q_{i}$-annuli, and $Z$-annuli.

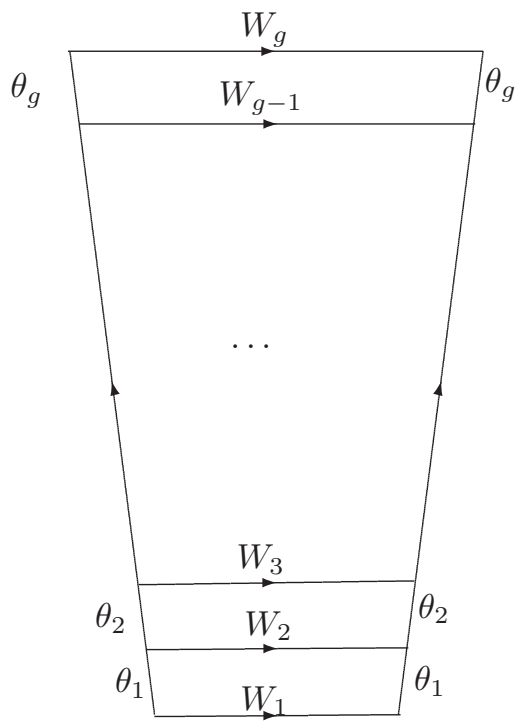

FiguRE 19. A computational sector.

Consider now an arbitrary computation $C=\left(W_{1}, W_{2}, \ldots, W_{g}\right)$ of an $S$-machine $\mathcal{S}$ with a history word $h$. With every $i=1, \ldots, g-1$ we associate the $\Theta$-band $\mathcal{T}_{i}$ with the boundary label $\theta_{i}^{-1} W_{i} \theta_{i} W_{i+1}^{-1}$ where $\theta_{i}$ is the $i$-letter in $h$. We can "concatenate" all these bands to obtain a van Kampen diagram with boundary of the form $\mathfrak{p}_{1}^{-1} \mathfrak{p}_{2} \mathfrak{p}_{3} \mathfrak{p}_{4}^{-1}$ where $\mathfrak{p}_{1}, \mathfrak{p}_{4}$ are labeled by $h, \operatorname{Lab}\left(\mathfrak{p}_{2}\right) \equiv W_{1}, \operatorname{Lab}\left(\mathfrak{p}_{4}\right) \equiv W_{g}$ (see Figure 19). This diagram is called a computational sector corresponding to the computation $C$.

In general by a sector we mean a reduced diagram $\Delta$ over the presentation of $\mathcal{S}$ with boundary divided into four parts, $\partial(\Delta)=\mathfrak{p}_{1}^{-1} \mathfrak{p}_{2} \mathfrak{p}_{3} \mathfrak{p}_{4}^{-1}$, such that the following properties hold:

- $\operatorname{Lab}\left(\mathfrak{p}_{1}\right), \operatorname{Lab}\left(\mathfrak{p}_{3}\right)$ are reduced group words in $\Theta^{+}$.

- $\operatorname{Lab}\left(\mathfrak{p}_{2}\right), \operatorname{Lab}\left(\mathfrak{p}_{4}\right)$ are admissible words.

The following lemma is essentially [29, Proposition 9.1].

Lemma 4.4. Every sector is combinatorially homotopic (even without insertions of cancelable cells) to a computational sector corresponding to a reduced computation connecting $\operatorname{Lab}\left(\mathfrak{p}_{2}\right)$ with $\operatorname{Lab}\left(\mathfrak{p}_{4}\right)$. The history of that computation is $\operatorname{Lab}\left(\mathfrak{p}_{1}\right) \equiv$ $\operatorname{Lab}\left(\mathfrak{p}_{3}\right)$. 


\subsection{Four $S$-machines.}

4.2.1. The $S$-machines $\mathcal{S}_{1}, \mathcal{S}_{2}$. By Lemma 3.5, the set of words $U(R)=\{U(\mu(r)) \mid$ $r \in R\}$ is recursive (see the notation in Remark [3.6). Let $\mathcal{S}_{1}$ be an $S$-machine recognizing that set of words and satisfying the conditions of Proposition 4.2

Let $\mathcal{S}_{2}$ be an $S$-machine recognizing the set $R$ written in the copy $\hat{A}$ of $A$ and satisfying the conditions of Proposition 4.2. By splitting the state letters (i.e. replacing in admissible words and in all rules a state letter $q$ by a product of new state letters $q^{\prime} q^{\prime \prime}$, we can assume that input bases (see Remark 4.1) of $\mathcal{S}_{1}$ and $\mathcal{S}_{2}$ are the same words and that the set of state letters of $\mathcal{S}_{i}$ has $m$ parts, $i=1,2$.

The tape alphabet of $\mathcal{S}_{1}$ is $Z\left(\mathcal{S}_{1}\right)=Z_{1} \cup \ldots \cup Z_{m-1}$ (we shall assume, without loss of generality, that $\left.Z_{1}=\emptyset\right)$; the state alphabet is $Q\left(\mathcal{S}_{1}\right)=Q_{1} \sqcup \ldots \sqcup Q_{m}$. The tape alphabet of $\mathcal{S}_{2}$ is $Z\left(\mathcal{S}_{2}\right)=\hat{Z}_{1} \cup \ldots \cup \hat{Z}_{m-1}$ (we shall assume that $\hat{Z}_{2}=\emptyset$ ); the state alphabet is $Q\left(\mathcal{S}_{2}\right)=\hat{Q}_{1} \sqcup \ldots \sqcup \hat{Q}_{m}$.

We shall assume that $A \cup B$ is contained in $Z_{2}$; there exists an injective map $\varepsilon$ from $A$ to $\hat{Z}_{1}$. The input word of $\mathcal{S}_{1}$ corresponding to a word $w$ in the alphabet $A \cup B$ has the form

$$
I_{1}(w) \equiv q_{1} q_{2} w q_{3} \ldots q_{m}
$$

(the word $w$ is between $q_{2}$ and $q_{3}$; there are no more tape letters in that word). The input word of $\mathcal{S}_{2}$ corresponding to a word $w$ in the alphabet $A$ is

$$
I_{2}(w) \equiv q_{1} \varepsilon(w) q_{2} q_{3} \ldots q_{m} .
$$

Thus the input bases of these $S$-machines are the same: $q_{1} \ldots q_{m}$. We shall also assume that $Q_{i} \cap \hat{Q}_{i}=\left\{q_{i}\right\}: Q_{i}$ and $\hat{Q}_{i}$ do not share letters except the state letters $q_{i}$ of the input bases. The stop words of $\mathcal{S}_{1}$ and $\mathcal{S}_{2}$ are denoted by $W_{0}\left(\mathcal{S}_{1}\right)$ and $W_{0}\left(\mathcal{S}_{2}\right)$, respectively.

Pick a number $N \geq 12$ and consider two new $S$-machines $\overline{\mathcal{S}}_{1}$ and $\overline{\mathcal{S}}_{2}$.

4.2.2. The $S$-machine $\overline{\mathcal{S}}_{1}$. For each $i=1, \ldots, 2 N$ let $Z^{(i)}$ be a disjoint copy of $Z$, and let $Q^{(i)}$ be a disjoint copy of $Q$. Then the tape alphabet of $\overline{\mathcal{S}}_{1}$ is

$$
\begin{aligned}
& Z\left(\overline{\mathcal{S}}_{1}\right)=\left(Z_{1}^{(1)} \cup \ldots \cup Z_{m-1}^{(1)}\right) \cup \emptyset \cup \emptyset \cup\left(Z_{m-1}^{(2)} \cup \ldots \cup Z_{1}^{(2)}\right) \cup \emptyset \cup \\
& \ldots \\
& \cup\left(Z_{1}^{(2 N-1)} \cup \ldots \cup Z_{m-1}^{(2 N-1)}\right) \cup \emptyset \cup \emptyset \cup\left(Z_{m-1}^{(2 N)} \cup \ldots \cup Z_{1}^{(2 N)}\right) \cup \emptyset ;
\end{aligned}
$$

the state alphabet is

$$
\begin{aligned}
& Q\left(\overline{\mathcal{S}}_{1}\right)=\left(Q_{1}^{(1)} \sqcup \ldots \sqcup Q_{m}^{(1)}\right) \sqcup\left\{k_{1}, \bar{k}_{1}\right\} \sqcup\left(Q_{m}^{(2)} \sqcup \ldots \sqcup Q_{1}^{(2)}\right) \sqcup\left\{t_{1}\right\} \sqcup \\
& \ldots \\
& \sqcup\left(Q_{1}^{(2 N-1)} \sqcup \ldots \sqcup Q_{m}^{(2 N-1)}\right) \sqcup\left\{k_{N}, \bar{k}_{N}\right\} \sqcup\left(Q_{m}^{(2 N)} \sqcup \ldots \sqcup Q_{1}^{(2 N)}\right) \sqcup\left\{t_{N}\right\} .
\end{aligned}
$$

The admissible words of the $S$-machine $\overline{\mathcal{S}}_{1}$ are described as follows. For every word $W$ in $Z\left(\mathcal{S}_{1}\right) \cup Q\left(\mathcal{S}_{1}\right)$ let $W^{(i)}$ be the corresponding copy of that word in the alphabet $Z\left(\mathcal{S}_{1}\right)^{(i)} \cup Q\left(\mathcal{S}_{1}\right)^{(i)}$. Also for every word $W$ let $\overleftarrow{W}$ be word $W$ read from right to left. If $W$ is an admissible word of $\mathcal{S}_{1}$ which is not an input word, then the corresponding admissible word of $\overline{\mathcal{S}}_{1}$ has the form

$$
W\left(\overline{\mathcal{S}}_{1}\right) \equiv W^{(1)} \bar{k}_{1} \overleftarrow{W}^{(2)} t_{1} W^{(3)} \bar{k}_{2} \overleftarrow{W}^{(4)} \ldots t_{N-1} W^{(2 N-1)} \bar{k}_{N} \overleftarrow{W}^{(2 N)} t_{N}
$$


If $I(u)$ is an input word, then the corresponding admissible word of $\overline{\mathcal{S}}_{1}$ is

$$
\bar{I}(u) \equiv I(u)^{(1)} k_{1} \overleftarrow{I(u)}^{(2)} t_{1} I(u)^{(3)} k_{2} \overleftarrow{I(u)^{(4)}} \ldots t_{N-1} I(u)^{(2 N-1)} k_{N} \overleftarrow{I(u)^{(2 N)}} t_{N}
$$

(in this case the $k$-letters are $k_{i}$ instead of $\bar{k}_{i}$ ).

The stop word of $\overline{\mathcal{S}}_{1}$, which will be called the first hub, is $W_{0}\left(\overline{\mathcal{S}}_{1}\right)$, the admissible word of $\overline{\mathcal{S}}_{1}$ corresponding to the stop word $W_{0}$ of $\mathcal{S}_{1}$. The rules of $\overline{\mathcal{S}}_{1}$ naturally correspond to the rules of $\mathcal{S}_{1}$ : if $\theta=\left[U_{1} \rightarrow V_{1}, \ldots, U_{n} \rightarrow V_{n}\right]$ is not the transition rule of $\mathcal{S}_{1}$ which we shall denote by $\tau_{1}$ (see Remark 4.1), then the corresponding rule $\bar{\theta}$ of $\overline{\mathcal{S}}_{1}$ is

$$
\bar{\theta}=\left[\begin{array}{l}
U_{1}^{(1)} \rightarrow V_{1}^{(1)}, \ldots, U_{n}^{(1)} \rightarrow V_{n}^{(1)}, k_{1} \rightarrow k_{1} \\
\overleftarrow{U_{n}}(2) \rightarrow \overleftarrow{V}_{n}^{(2)}, \ldots, \overleftarrow{U}_{1}^{(2)} \rightarrow \overleftarrow{V}_{1}^{(2)}, t_{1} \rightarrow t_{1} \\
\ldots \\
U_{1}^{(2 N-1)} \rightarrow V_{1}^{(2 N-1)}, \ldots, U_{n}^{(2 N-1)} \rightarrow V_{n}^{(2 N-1)}, k_{N} \rightarrow k_{N} \\
\overleftarrow{U_{n}}(2 N) \rightarrow \overleftarrow{V}_{n}^{(2 N)}, \ldots, \overleftarrow{U}_{1}^{(2 N)} \rightarrow \overleftarrow{V}_{1}^{(2 N)}, t_{N} \rightarrow t_{N}
\end{array}\right]
$$

Thus the rule $\bar{\theta}$ simultaneously executes copies of the rule $\theta$ on all $\left(Q_{1}^{(i)}, Q_{m}^{(i)}\right)$ subwords of an admissible word, $i=1, \ldots, 2 N$. Essentially $\overline{\mathcal{S}}_{1}$ runs simultaneously $N$ copies of $\mathcal{S}_{1}$, which we denote by $\mathcal{S}_{1}^{(2 i-1)}, i=1, \ldots, N$, and $N$ copies of the mirror images $\overleftarrow{\mathcal{S}}_{1}^{(2 i)}$ of $\mathcal{S}_{1}, i=1, \ldots, N$

If $\theta$ is the transition rule $\tau_{1}=\left[q_{1} \ldots q_{m} \rightarrow \tilde{q}_{1} \ldots \tilde{q}_{m}\right]$, then the corresponding transition rule $\overline{\mathcal{S}}_{1}$ is

$$
\bar{\tau}_{1}=\left[\begin{array}{l}
q_{1}^{(1)} \ldots q_{m}^{(1)} \rightarrow \tilde{q}_{1}^{(1)} \ldots \tilde{q}_{m}^{(1)}, k_{1} \rightarrow \bar{k}_{1} \\
q_{m}^{(2)} \ldots q_{1}^{(2)} \rightarrow \tilde{q}_{m}^{(2)} \ldots \tilde{q}_{1}^{(2)}, t_{1} \rightarrow t_{1} \\
\ldots \\
q_{1}^{(2 N-1)} \ldots q_{m}^{(2 N-1)} \rightarrow \tilde{q}_{1}^{(2 N-1)} \ldots \tilde{q}_{m}^{(2 N-1)}, k_{N} \rightarrow \bar{k}_{N} \\
q_{m}^{(2 N)} \ldots q_{1}^{(2 N)} \rightarrow \tilde{q}_{m}^{(2 N)} \ldots \tilde{q}_{1}^{(2 N)}, t_{N} \rightarrow t_{N}
\end{array}\right]
$$

(that is, the rule changes all $q_{j}^{(i)}$ to $\tilde{q}_{j}^{(i)}$ and all $k_{i}$ to $\bar{k}_{i}$ ).

4.2.3. The $S$-machine $\overline{\mathcal{S}}_{2}$. This machine is constructed using $\mathcal{S}_{2}$ in a way similar to how $\overline{\mathcal{S}}_{1}$ is constructed from $\mathcal{S}_{1}$ except that the set of rules is constructed somewhat differently.

For each $i=1, \ldots, 2 N$ let $\hat{Z}^{(i)}$ be a disjoint copy of $\hat{Z}$, and let $\hat{Q}^{(i)}$ be a disjoint copy of $\hat{Q}$. We identify $\hat{Z}^{(1)}$ with $\hat{Z}$. Then the tape alphabet of $\overline{\mathcal{S}}_{2}$ is

$$
\begin{aligned}
& Z\left(\overline{\mathcal{S}}_{2}\right)=\underbrace{\emptyset \cup \ldots \cup \emptyset}_{m-1} \cup \emptyset \cup \emptyset \cup\left(\hat{Z}_{m-1}^{(2)} \cup \ldots \cup \hat{Z}_{1}^{(2)}\right) \cup \emptyset \\
& \cup\left(\hat{Z}_{1}^{(3)} \cup \ldots \cup \hat{Z}_{m-1}^{(3)}\right) \cup \emptyset \cup \emptyset \cup\left(\hat{Z}_{m-1}^{(4)} \cup \ldots \cup \hat{Z}_{1}^{(4)}\right) \cup \emptyset \\
& \ldots \\
& \sqcup\left(\hat{Z}_{1}^{(2 N-1)} \cup \ldots \cup \hat{Z}_{m-1}^{(2 N-1)}\right) \cup \emptyset \sqcup \emptyset \cup\left(\hat{Z}_{m-1}^{(2 N)} \cup \ldots \cup \hat{Z}_{1}^{(2 N)}\right) \cup \emptyset ;
\end{aligned}
$$

the state alphabet is

$$
\begin{aligned}
& Q\left(\overline{\mathcal{S}}_{2}\right)=\left(\hat{Q}_{1}^{(1)} \sqcup \ldots \sqcup \hat{Q}_{m}^{(1)}\right) \sqcup\left\{k_{1}, \hat{k}_{1}\right\} \sqcup\left(\hat{Q}_{m}^{(2)} \sqcup \ldots \sqcup \hat{Q}_{1}^{(2)}\right) \sqcup\left\{t_{1}\right\} \sqcup \\
& \ldots \\
& \sqcup\left(\hat{Q}_{1}^{(2 N-1)} \sqcup \ldots \sqcup \hat{Q}_{m}^{(2 N-1)}\right) \sqcup\left\{k_{N}, \hat{k}_{N}\right\} \sqcup\left(\hat{Q}_{m}^{(2 N)} \sqcup \ldots \sqcup \hat{Q}_{1}^{(2 N)}\right) \sqcup\left\{t_{N}\right\} .
\end{aligned}
$$

The description of the admissible words of $\overline{\mathcal{S}}_{2}$ is the following. For every word $W$ in the alphabet $\hat{Z} \cup \hat{Q}$ let $W^{(i)}$ be the corresponding copy of that word in the alphabet 
$\hat{Z}^{(i)} \cup \hat{Q}^{(i)}$. For every word $W$ in the alphabet $\hat{Z} \cup \hat{Q}$ let $q(W) \equiv W_{\hat{Q}}$ be the word $W$ with all letters from $\hat{Z}$ deleted (the projection of $W$ onto $\hat{Q}$ ). If $W$ is an admissible word of $\mathcal{S}_{2}$ but not an input word, then the corresponding admissible word of $\overline{\mathcal{S}}_{2}$ has the form

$$
W\left(\overline{\mathcal{S}}_{2}\right) \equiv q\left(W^{(1)}\right) \hat{k}_{1} \overleftarrow{W}^{(2)} t_{1} W^{(3)} \hat{k}_{2} \overleftarrow{W}^{(4)} \ldots t_{N-1} W^{(2 N-1)} \hat{k}_{N} \overleftarrow{W}^{(2 N)} t_{N}
$$

The admissible word $\bar{I}_{2}(u)$ corresponding to the input word $I_{2}(u)$ of $\mathcal{S}_{2}$ is

$$
q\left(I_{2}(u)\right) k_{1}{\overleftarrow{I_{2}(u)}}^{(2)} t_{1} I_{2}(u)^{(3)} k_{2}{\overleftarrow{I_{2}(u)}}^{(4)} \ldots t_{N-1} I_{2}(u)^{(2 N-1)} k_{N} \overleftarrow{I_{2}(u)^{(2 N)}} t_{N}
$$

The stop word of $\overline{\mathcal{S}}_{2}$ is the word $W_{0}\left(\overline{\mathcal{S}}_{2}\right)$ which will be called the second hub.

The rules of $\overline{\mathcal{S}}_{2}$ correspond to rules of $\mathcal{S}_{2}$. If $\theta=\left[U_{1} \rightarrow V_{1}, \ldots, U_{n} \rightarrow V_{n}\right]$ is a rule of $\mathcal{S}_{2}$ but not the transition rule, which we shall denote $\tau_{2}$, then the corresponding rule $\bar{\theta}$ of $\overline{\mathcal{S}}_{2}$ is (compare with (4.1))

$$
\bar{\theta}=\left[\begin{array}{l}
q\left(U_{1}^{(1)}\right) \stackrel{\ell}{\rightarrow} q\left(V_{1}^{(1)}\right), \ldots, q\left(U_{n}^{(1)}\right) \stackrel{\ell}{\rightarrow} q\left(V_{n}^{(1)}\right), \hat{k}_{1} \rightarrow \hat{k}_{1}, \\
\overleftarrow{U}_{n}^{(2)} \rightarrow \overleftarrow{V}_{n}^{(2)}, \ldots, \overleftarrow{U}_{1}^{(2)} \rightarrow \overleftarrow{V}_{1}^{(2)}, t_{1} \rightarrow t_{1} \\
\ldots \\
U_{1}^{(2 N-1)} \rightarrow V_{1}^{(2 N-1)}, \ldots, U_{n}^{(2 N-1)} \rightarrow V_{n}^{(2 N-1)}, \hat{k}_{N} \rightarrow \hat{k}_{N} \\
\overleftarrow{U}_{m}^{(2 N)} \rightarrow \overleftarrow{V}_{m}^{(2 N)}, \ldots, \overleftarrow{U}_{1}^{(2 N)} \rightarrow \overleftarrow{V}_{1}^{(2 N)}, t_{N} \rightarrow t_{N}
\end{array}\right]
$$

Thus the $S$-machine $\overline{\mathcal{S}}_{2}$ does not insert of delete any tape letters in the $\left(Q_{1}^{(1)}, Q_{m}^{(2)}\right)$ subwords of admissible words. If $\theta$ is the transition rule $\tau_{2}=\left[q_{1} \ldots q_{m} \rightarrow \tilde{q}_{1} \ldots \tilde{q}_{m}\right]$, then the corresponding transition rule of $\overline{\mathcal{S}}_{2}$ is

$$
\bar{\tau}_{2}=\left[\begin{array}{l}
q_{1}^{(1)} \ldots q_{m}^{(1)} \rightarrow \tilde{q}_{1}^{(1)} \ldots \tilde{q}_{m}^{(1)}, k_{1} \rightarrow \hat{k}_{1}, \\
q_{m}^{(2)} \ldots q_{1}^{(2)} \rightarrow \tilde{q}_{m}^{(2)} \ldots \tilde{q}_{1}^{(2)}, t_{1} \rightarrow t_{1}, \\
\ldots \\
q_{1}^{(2 N-1)} \ldots q_{m}^{(2 N-1)} \rightarrow \tilde{q}_{1}^{(2 N-1)} \ldots \tilde{q}_{m}^{(2 N-1)}, k_{N} \rightarrow \hat{k}_{N}, \\
q_{m}^{(2 N)} \ldots q_{1}^{(2 N)} \rightarrow \tilde{q}_{m}^{(2 N)} \ldots \tilde{q}_{1}^{(2 N)}, t_{N} \rightarrow t_{N}
\end{array}\right] .
$$

4.3. The composition of the $S$-machines and the auxiliary group. Let $A$ be the generating set of our group $\Gamma$. Let $A \cup B \cup X \cup Y$ be the generating set of $H(A)$. For every $i=1, \ldots, 2 N$ we define copies $A^{(i)}, B^{(i)}, X^{(i)}, Y^{(i)}$ of the sets $A, B, X, Y$, and the generating set of the group $H^{(i)}(A)$. The relations of $H^{(i)}(A)$ are the relations of $H\left(A^{(i)}\right)$ if $i$ is odd. If $i$ is even, then the relations of $H^{(i)}(A)$ are obtained from the relations of $H\left(A^{(i)}\right)$ by reading them from right to left. Clearly, there exists an isomorphism from $H^{(i)}(A)$ onto $H\left(A^{(i)}\right)$ for even $i$ given by the map $z \mapsto z^{-1}$.

We add the generators $X^{(i)}, Y^{(i)}$ and relators of all groups $H^{(i)}(A)$ to the already introduced generators and relators. In addition we shall need all relations of the form

$$
z^{(2 i-1)}\left(q_{3}^{(2 i-1)} \ldots q_{m}^{(2 i-1)} k_{i} q_{m}^{(2 i)} \ldots q_{3}^{(2 i)}\right)=\left(q_{3}^{(2 i-1)} \ldots q_{m}^{(2 i-1)} k_{i} q_{m}^{(2 i)} \ldots q_{3}^{(2 i)}\right) z^{(2 i)}
$$

for all $z \in X \cup Y$, and all relations of the form

$$
\varepsilon(a)^{(2 i-1)} q_{2}^{(2 i-1)}=q_{2}^{(2 i-1)} u_{a}^{(2 i-1)} v_{a}^{(2 i-1)}
$$


for all $a \in A, i=1, \ldots, N$, where $u_{a} v_{a}=\mu(a)$ is the generator of the subgroup $E$ corresponding to $a \in A$

$$
q_{2}^{(2 i)} \varepsilon(a)^{(2 i)}={\overleftarrow{v_{a}}}^{(2 i)}{\overleftarrow{u_{a}}}^{(2 i)} q_{2}^{(2 i)}
$$

for all $a \in A, i=1, \ldots, N$.

\subsection{The group $\mathcal{G}$.}

Definition 4.5. The group $\mathcal{G}$ is the group given by the defining relations from Sections 4.2.2, 4.2.3, 4.3 and the two hub relations $W_{0}\left(\overline{\mathcal{S}}_{1}\right)=1$ and $W_{0}\left(\overline{\mathcal{S}}_{2}\right)=1$. Thus the finite presentation of $\mathcal{G}$ consists of

- defining relations of $\overline{\mathcal{S}}_{i}, i=1,2$ (called the $\overline{\mathcal{S}}_{i}$-relations),

- the defining relations of $H^{(i)}(A)$ (called the $H^{(i)}(A)$-relations),

- the relations (4.4) (called the gluing $k_{i}$-relations),

- the relations (4.5) and (4.6) (called the conjugacy $q_{2}^{(i)}$-relations),

- the two hub relations

$$
W_{0}\left(\overline{\mathcal{S}}_{1}\right)=1, \quad W_{0}\left(\overline{\mathcal{S}}_{2}\right)=1 .
$$

The role played by each of these relations will be clear in Section 5.1

\section{The MAIN RESUlT}

We are going to prove the following theorem. Recall that $\varepsilon$ is an injective map from $A$ to $\hat{Z}_{1}$. We shall identify $\hat{Z}$ with $\hat{Z}^{(1)} \subset \mathcal{G}$.

Theorem 5.1. (1) The map $\varepsilon: A \rightarrow \mathcal{G}$ extends to a homomorphism $\Gamma \rightarrow \mathcal{G}$ which will be denoted by $\varepsilon$ as well.

(2) The homomorphism $\varepsilon$ is injective.

(3) If the presentation $\langle A \mid R\rangle$ of $\Gamma$ is combinatorially aspherical, then the (finite) presentation of $\mathcal{G}$ is combinatorially aspherical.

5.1. The map $\varepsilon$ is a homomorphism. Part (1) of Theorem 5.1 is given by the following lemma.

Lemma 5.2. For every $r \in R, \varepsilon(r)=1$ in $\mathcal{G}$.

Proof. To prove the lemma, we build a van Kampen diagram for the relation $\varepsilon(r)=$ 1 for every $r \in R$. That van Kampen diagram will be denoted by $\Psi(r)$ and will be called standard. Since this diagram is important, we will describe a step-by-step procedure of constructing it.

Step 1. Using $H(A)$-relations and conjugacy relations, construct a van Kampen diagram for the equality $q_{2}^{-1} \varepsilon(r) q_{2}=U(r) V(r)$ (i.e. with the boundary label $q_{2}^{-1} \varepsilon(r) q_{2}(U(r) V(r))^{-1}$; see Figure 201).

Step 2. Take $N$ copies of the diagram from Step 1 and $N$ copies of the mirror image of this diagram and glue them using the gluing relations (see Figure 21). For the sake of brevity, we did not include upper indexes of letters; the upper indexes of letters to the left of $k_{i}$ are $2 i-1$, and the upper indexes of letters to the right of $k_{i}$ are $2 i$. 


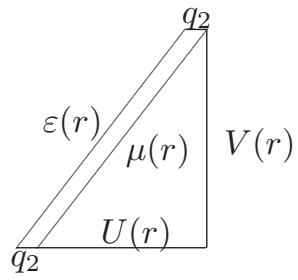

Figure 20. The van Kampen diagram for the equality $q_{2}^{-1} \varepsilon(r) q_{2}=U(r) V(r)$.

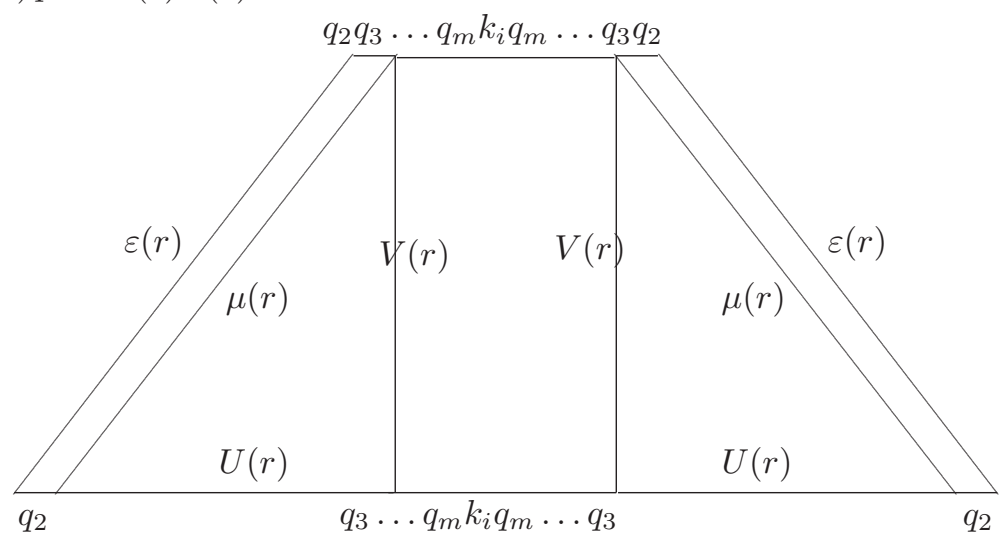

Figure 21. Gluing a copy of the diagram on Figure 20 and a copy of its mirror image.

Step 3. The machine $\bar{S}_{1}$ accepts $I_{1}(r)$. Therefore there exists a computational sector (Figure 19] see [29]) whose upper side is labeled by $I_{1}(r)$, the lower side is labeled by the stop word $W_{0}\left(\bar{S}_{1}\right)$, and the left and right sides are labeled by the same word, the history $h$ of accepting computation. Gluing together the left and right sides and inserting the first hub into the hole of the resulting annular diagram gives us the first disc. Similarly $I_{2}(r)$ is accepted by $\bar{S}_{2}$ and we can form the second disc (see Figure 22).
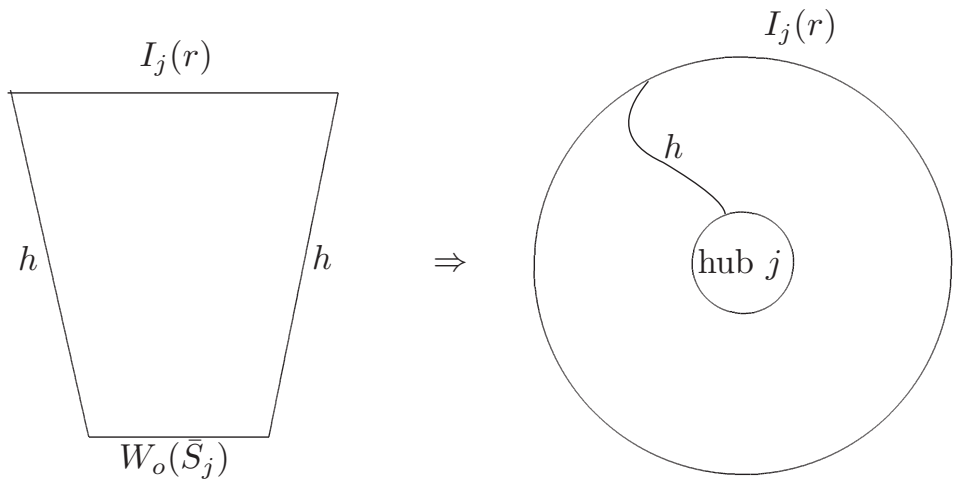

Figure 22. From a computational sector to a disc. Here $j=1,2$, and $h$ is the history of accepting computation. 
Step 4. Glue the diagrams obtained in Step 2 to the boundary of disc 1 and obtain a new van Kampen diagram. Let us denote it $\Delta_{1}$ (see Figure 23).

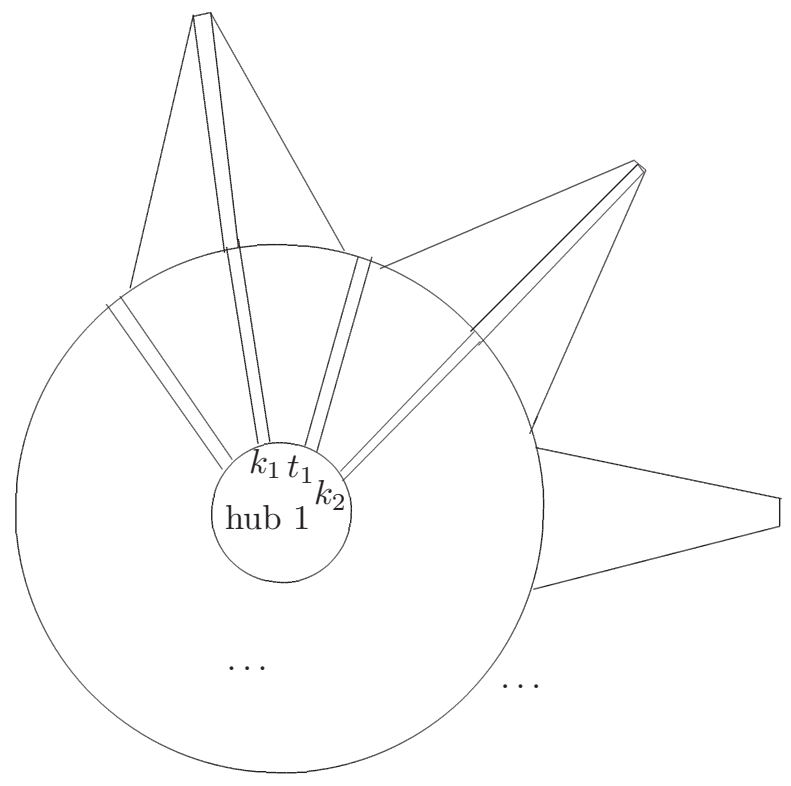

Figure 23. Gluing diagrams from Step 2 to disc 1.

Step 5 . The word written on the boundary of the diagram $\Delta_{1}$ obtained in Step 4 is equal to

$$
\begin{aligned}
& q_{1}^{(1)} \varepsilon(r)^{(1)} q_{2}^{(1)} \ldots q_{m}^{(1)} k_{1} q_{m}^{(2)} \ldots q_{2}^{(2)} \overleftarrow{\varepsilon(r)}{ }^{(2)} t_{1} \\
& \ldots \\
& q_{1}^{(2 N-1)} \varepsilon(r)^{(2 N-1)} q_{2}^{(2 N-1)} \ldots q_{m}^{(2 N-1)} k_{N} q_{m}^{(2 N)} \ldots q_{2}^{(2 N)} \overleftarrow{\varepsilon(r)}{ }^{(2 N)} t_{N} .
\end{aligned}
$$

This word can be obtained from the word $\bar{I}_{2}(r)$ by inserting the word $\varepsilon(r)^{(1)} \equiv \varepsilon(r)$ between $q_{1}^{(1)}$ and $q_{2}^{(1)}$.

Let us identify the initial and terminal vertices of the subpath of the boundary $\partial(\Delta)$ labeled by $\varepsilon(r)$. The result is an annular diagram with a boundary of the hole labeled by $\varepsilon(r)$ and the outside boundary labeled by $\bar{I}_{1}(r)$ (see Figure 24). Let us call the new (annular) diagram $\Delta_{1}^{\prime}$.

Now consider a sphere $S^{2}$. Draw the diagram $\Delta_{1}^{\prime}$ on the sphere so that the outer boundary of $\Delta^{\prime}$ coincides with the equator and the hub is on the north pole. Since the labels of the outer boundary of the diagram $\Delta^{\prime}$ and of the boundary of disc 2 are the same, we can draw disc 2 on the southern hemisphere of our sphere, so that the second hub is at the south pole and the boundary of disc 2 also coincides with the equator. The result is a van Kampen diagram over the presentation of $\mathcal{G}$ drawn on the sphere with a hole (i.e. a disc) and the label of the boundary of that diagram is $\varepsilon(r)$ as required. 

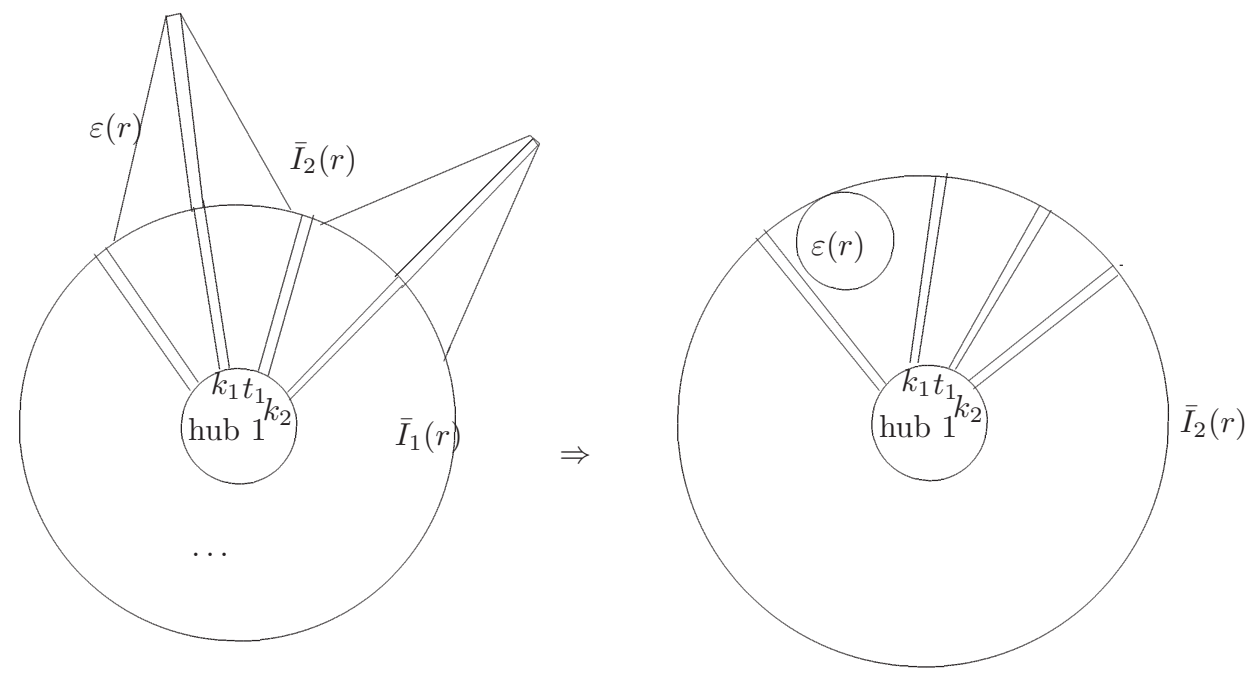

FiguRE 24. The northern hemisphere (with a hole labeled by $\varepsilon(r)$ ).

Notation 5.3. Let $\Psi(r)$ be the diagram constructed in the proof of Lemma 5.2. It contains two hubs $\pi_{1}$ and $\pi_{2}$ (located at the north and the south poles) connected by $t_{1^{-}}, \ldots, t_{N^{-}}$and $k_{1^{-}}, \ldots, k_{N^{-}}$bands (meridians). It is convenient to make the notation independent on how we enumerate the hubs in $\Psi(r)$, so we assume that $\pi_{1}$ is a $j$-th hub and $\pi_{2}$ is a $(3-j$ )-th hub (for some $j=1,2)$. If $i<N$, then the subdiagram bounded by the $t_{i}$-band and the $k_{i+1}$-band and $\partial\left(\mathcal{T}_{1}\right), \partial\left(\mathcal{T}_{2}\right)$ is denoted by $\Psi_{i}(r)$. The subdiagram $\Psi_{i}(r)$ contains two maximal transition $\theta$-bands $\mathcal{A}_{1}, \mathcal{A}_{2}$. The medians of these bands divide $\Psi_{i}(r)$ into three parts $\Psi_{i}^{\prime}(r), \Psi_{i}^{\prime \prime}(r), \Psi_{i}^{\prime \prime \prime}(r)$ counting from $\pi_{1}$ to $\pi_{2}$. The first and the third subdiagrams are computational sectors corresponding to the computation of $\mathcal{S}_{j}^{(2 i+1)}$ accepting $I_{j}^{(i+1)}(U(r))$ and the computation of $\mathcal{S}_{3-j}^{(2 i+1)}$ accepting $I_{3-j}^{(i+1)}(r)$, respectively, where $j=1$ or 2 (recall that $i<N)$. The union of $\Psi_{i}^{\prime}(r) \cup \mathcal{A}_{1} \cup \Psi^{\prime \prime}(r) \cup \mathcal{A}_{2}$ is denoted by $\Phi_{i}(r)$. The complement of that subdiagram in $\Psi(r)$ will be denoted by $\bar{\Phi}_{i}(r)$.

5.2. Expanded presentation of $\mathcal{G}$. Let us denote the finite presentation of $\mathcal{G}$ given in Section 4.4 by $\mathcal{P}$ and the presentation obtained from $\mathcal{P}$ by adding all $\Gamma$-relations $\varepsilon(r), r \in R$, by $\mathcal{P}^{\prime}$.

Notation 5.4. We shall study diagrams over $\mathcal{P}$ and $\mathcal{P}^{\prime}$. We say that two diagrams $\Delta_{1}, \Delta_{2}$ over $\mathcal{P}^{\prime}$ are combinatorially $\mathcal{P}$-homotopic if one can transform $\Delta_{1}$ to $\Delta_{2}$ using diamond moves and insertion and deletion of cancelable cells corresponding to relations from $\mathcal{P}$. If $\Delta$ is a diagram over $\mathcal{P}$, we can consider the following bands in $\Delta$ :

- $\theta$-bands, $\theta \in \bar{\Theta}_{1}^{+} \cup \bar{\Theta}_{2}^{+}$, consisting of $(\theta, a),(\theta, q)$-, and $(\theta, k)$-cells. These bands can start and end on the boundary of $\Delta$.

- $Q_{i}^{(j)}$-bands, $i=1,3,4, \ldots, m, j=1, \ldots, 2 N$, consisting of $(Q, \Theta)$-cells and gluing $k_{j}$-cells $(i=3, \ldots, m)$. These bands can start (end) on the hub cells and on the boundary of $\Delta$. 
- $Q_{2}^{(j)}$-bands, $j=1, \ldots, 2 N$, consisting of $(Q, \Theta)$-cells and conjugacy $q_{2}^{(i)}$ cells. These bands can start (end) on the hub cells and on the boundary of $\Delta$.

- $k_{i}$-bands consisting of $\left(k_{i}, \Theta\right)$-cells and gluing $k_{i}$-cells. These bands can start (end) on the hub cells and on the boundary of $\Delta$.

- $t_{i}$-bands consisting of $\left(t_{i}, \Theta\right)$-cells. These bands can start (end) on the hub cells and on the boundary of $\Delta$.

- $Z$-bands consisting of $(Z, \Theta)$-cells, $\left(A^{(i)}, X^{(i)}\right)$-cells, and $\left(A^{(i)}, Y^{(i)}\right)$-cells. These bands can start (end) on the boundary of $\Delta$ or on a $(Q, \theta)$-cell or on a conjugacy $q_{2}^{(i)}$-cell.

- $X^{(i)}$-bands consisting of $\left(A^{(i)}, X^{(i)}\right)$ - and $\left(B^{(i)}, X^{(i)}\right)$-cells. These bands can start (end) on the boundary of $\Delta$ or on the boundary of a $k_{i}$-gluing cell or on the boundary of a $q_{2}^{(i)}$-conjugacy cell.

Notation 5.5. Let $\Delta$ be a reduced diagram having two hubs $\pi_{1}, \pi_{2}$ connected by a $t_{i}$-band $\mathcal{X}_{1}$ and a $k_{i+1}$-band $\mathcal{X}_{2}$ where $1 \leq i<N$. Suppose that $\Psi$ is bounded by a side of $\mathcal{X}_{1}$, a side of $\mathcal{X}_{2}$, and parts of $\partial \pi_{1}, \partial \pi_{2}$ and does not contain hubs except for $\pi_{1}, \pi_{2}$. We shall call such a diagram a 2-hub $i$-diagram. The subdiagram obtained from $\Delta$ by removing $\pi_{1}, \pi_{2}, \mathcal{X}_{1}, \mathcal{X}_{2}$ is denoted by $\Delta^{\prime}$ and is called the inside subdiagram of $\Delta$. The band $\mathcal{X}_{2}$ consists of $\left(k_{i+1}, \theta\right)$-cells, transition cells, and gluing $k_{i+1}$-cells. The number of transition cells in $\mathcal{K}$ will be called the complexity of $\Delta$.

Note that the diagram $\Psi_{i}(r)$ from Notation 5.3 is a 2-hub $i$-diagram of complexity 2 .

Notation 5.6. Let us define several groups given by subpresentations of $\mathcal{P}^{\prime}$.

1. For every $j=2, \ldots, N$, let $K_{1}^{(j)}$ be the group given by all the relations of $H^{(2 j-1)}(A)$ and the conjugacy $q_{2}^{(2 j-1)}$-relations.

2. For every $j=1, \ldots, N$ let $K_{2}^{(j)}$ be the group given by all the relations of $\overleftarrow{H}^{(2 i)}(A)$ and the conjugacy $q_{2}^{(2 j)}$-relations.

3. For every $j=2, \ldots, N$ let $K^{(j)}$ be the group given by all the relations of $K_{i}^{(j)}, i=1,2$, and the gluing $k_{j}$-relations.

4. Let $K_{1}^{(1)}$ be the group given by all the relations of $H^{(1)}(A)$, defining relations of $\varepsilon(\Gamma)$, and the conjugacy $q_{2}^{(1)}$-relations.

5. Let $K^{(1)}$ be the group given by all the relations of $K_{1}^{(1)}$, relations of $K_{2}^{(1)}$, and the gluing $k_{1}$-relation.

6. Let $\mathcal{K}$ be the free product of all $K^{(i)}, i=1, \ldots, N$.

Clearly, the presentation of $\mathcal{K}$ is a subpresentation of $\mathcal{P}^{\prime}$.

Lemma 5.7. For every $j=2, \ldots, N$, the set $X^{(2 j-1)} \cup Y^{(2 j-1)}$ freely generates a free subgroup in the group $K_{1}^{(j)}$.

Proof. Indeed, that group is an HNN-extension of the free product of $H^{(2 j-1)} *$ $\left\langle\varepsilon(A)^{(2 j-1)}\right\rangle$ (the second factor is a free group generated by $\varepsilon(A)^{(2 j-1)}$ ) with free letter $q_{2}^{(2 j-1)}$ and associated subgroups generated by sets $\varepsilon(A)^{(2 j-1)}$ and $\mu(A)^{(2 j-1)}$, respectively. Both sets freely generate free subgroups in the free product by the definitions of $\varepsilon$ and $\mu$.

The following lemma is proved in the same way as Lemma 5.7 
Lemma 5.8. For every $j=1, \ldots, N$, the set $X^{(2 j)} \cup Y^{(2 j)}$ freely generates a free subgroup in the group $K_{2}^{(j)}$.

Lemma 5.9. For every $j=2, \ldots, N, K^{(j)}$ is constructed as follows. First take the $H N N$-extension $\hat{K}^{(j)}$ of the free product of $K_{1}^{(j)} * K_{2}^{(1)}$ with free letter $\hat{k}_{j}$ and free associated subgroups generated by $X^{(2 j-1)} \cup Y^{(2 j-1)}$ and $X^{(2 j)} \cup Y^{(2 j)}$, respectively. Then $K^{(j)}$ is an amalgamated product of $\hat{K}^{(j)}$ and the free group freely generated by $q_{3}^{(2 j-1)}, \ldots, q_{m}^{(2 j-1)}, k_{m}, q_{3}^{(2 j)}, \ldots, q_{m}^{(2 j)}$ with cyclic associated subgroups generated by $\hat{k}_{j}$ and $q_{3}^{(2 j-1)} \ldots q_{m}^{(2 j-1)} k_{j} q_{m}^{(2 j)} \ldots q_{3}^{(2 j)}$, respectively.

Proof. This immediately follows from the definition of $K^{(j)}$ and Lemmas 5.7 and 5.8 .

Lemma 5.10. The group $K^{(1)}$ is constructed as follows. First take the HNNextension $\hat{K}^{(1)}$ of the free product of $H^{\prime}(\Gamma) * K_{2}^{(1)}$ with free letter $\hat{k}_{1}$ and free associated subgroups freely generated by $X^{(1)} \cup Y^{(1)}$ and $X^{(2)} \cup Y^{(2)}$, respectively. Then $K^{(1)}$ is an amalgamated product of $\hat{K}^{(1)}$ and the free group freely generated by $q_{3}^{(1)}, \ldots, q_{m}^{(1)}, k_{1}, q_{3}^{(2)}, \ldots, q_{m}^{(2)}$ with cyclic associated subgroups generated by $\hat{k}_{1}$ and $q_{3}^{(1)} \ldots q_{m}^{(1)} k_{1} q_{m}^{(2)} \ldots q_{3}^{(2)}$, respectively.

Proof. Indeed, the group $K_{1}^{(1)}$ is clearly isomorphic to $H^{\prime}(\Gamma)$. Then by Lemma 3.19. the group $H(A) / \mathcal{N}$ naturally embeds into $K_{1}^{(1)}$ where $\mathcal{N}$ is the normal subgroup of $H(A)$ generated by $\mu(R)$ (see Lemma 3.19). This, the second equality of Lemma 3.16. and Lemma 3.21 imply that the sets $X^{(1)} \cup Y^{(1)}$ and $X^{(2)} \cup Y^{(2)}$ freely generate free subgroups of $K_{1}^{(1)}$ and $K_{2}^{(2)}$, respectively. Then it is clear that the presentation of $\hat{K}^{(1)}$ is the standard presentation of the HNN-extension and the presentation of $K^{(1)}$ is the standard presentation of an amalgamated product.

Lemma 5.11. The set $\bigcup\left(A^{(j)} \cup B^{(j)}\right)$ generates a free subgroup in $\mathcal{K}$.

Proof. This immediately follows from Lemmas 3.3, 3.16, and 3.19.

Lemma 5.12. Let $\Delta$ be a reduced diagram over $\mathcal{P}^{\prime}$ without hubs. Then it does not have $\Theta$-annuli.

Proof. Suppose that $\Delta$ is a counterexample with the smallest number of cells and that $\mathcal{T}$ is a $\Theta$-annulus in $\Delta$. Let $\Delta^{\prime}$ be the inside subdiagram of $\mathcal{T}$.

Since $\partial \Delta^{\prime}$ does not contain $\Theta$-edges, $\Delta^{\prime}$ does not have any cells corresponding to the defining relations of $\overline{\mathcal{S}}_{i}, i=1,2$ (because $\Delta^{\prime}$ has fewer cells than $\Delta$ ). Therefore $\Delta^{\prime}$ is a diagram over the presentation of the group $\mathcal{K}$ (since $\Delta$ does not contain hubs and all other relations have been ruled out). Note that different cells of $\mathcal{T}$ cannot have non- $\Theta$-edges in common; otherwise they cancel. Hence every cell in $\mathcal{T}$ has a common edge with a cell corresponding to a relation of $\mathcal{K}$. Therefore $\mathcal{T}$ corresponds to a transition rule of $\mathcal{S}_{1}$ or of $\mathcal{S}_{2}$.

In the first case the boundary label of $\Delta^{\prime}$ is a word in

$$
\bigcup_{i=1}^{2 N}(A \cup B)^{i} \cup \bigcup_{i=1}^{2 N}\left\{q_{1}^{(i)}, \ldots, q_{m}^{(i)}\right\} \cup\left\{k_{1}, \ldots, k_{N}\right\} .
$$

In the second case it is a word in

$$
\bigcup_{i=2}^{2 N} \varepsilon(A)^{(i)} \cup \bigcup_{i=1}^{2 N}\left\{q_{1}^{(i)}, \ldots, q_{m}^{(i)}\right\} \cup\left\{k_{1}, \ldots, k_{N}\right\} .
$$


We claim that both sets freely generate free subgroups in $\mathcal{K}$. Indeed, since $\mathcal{K}$ is a free product, it is enough to show that the set

$$
A^{(2 i-1)} \cup B^{(2 i-1)} \cup\left\{q_{1}^{(2 i-1)}, \ldots, q_{m}^{(2 i-1)}\right\} \cup\left\{k_{i}\right\} \cup A^{(2 i)} \cup B^{(2 i)} \cup\left\{q_{1}^{(2 i)}, \ldots, q_{m}^{(2 i)}\right\},
$$

the set

$$
\varepsilon(A)^{(2 i-1)} \cup\left\{q_{1}^{(2 i-1)}, \ldots, q_{m}^{(2 i-1)}\right\} \cup\left\{k_{i}\right\} \cup\left\{q_{1}^{(2 i)}, \ldots, q_{m}^{(2 i)}\right\} \cup \varepsilon(A)^{(2 i)}, i \geq 2,
$$

and the set

$$
\left\{q_{1}^{(1)}, \ldots, q_{m}^{(1)}\right\} \cup\left\{k_{1}\right\} \cup\left\{q_{1}^{(2)}, \ldots, q_{m}^{(2)}\right\} \cup \varepsilon(A)^{(2)}
$$

freely generate a free subgroup in the corresponding $K^{(i)}, i=1, \ldots, N$. For the set (5.1) it follows the representation of $K^{(i)}$ as an amalgamated product (Lemmas 5.9 and 5.10) and Lemma 3.3 if $i \geq 2$ and Lemmas 3.3 and 3.16 if $i=1$. For the sets (5.2) and (5.3) it follows from the representation of $K^{(i)}$ as an amalgamated product, the fact that the subgroup $E$ of $H(A)$ is freely generated by $\mu(a), a \in A$ (by construction), and the fact that the set $\varepsilon(A)^{(j)}$ is conjugated to the set $\mu(A)^{(j)}$ in $K_{i}^{(j)}$ where $i=1, j \geq 2$ or $i=2, j \geq 1$ by $q_{2}^{(j)}$.

The claim shows that the inner boundary of $\mathcal{T}$ has freely trivial label; hence $\mathcal{T}$ has two cells that cancel, a contradiction.

Lemma 5.13. Let $\Delta$ be a reduced diagram over $\mathcal{P}^{\prime}$ without hubs and $\theta$-edges. Then $\Delta$ does not have $k_{1}$-annuli.

Proof. Suppose that $\Delta$ contains a $k_{1}$-annulus $\mathcal{T}$. Let $\Delta^{\prime}$ be the inside diagram of that annulus. Note that $\partial \Delta^{\prime}$ consists of $X^{(1)} \cup Y^{(1)}$-edges. Since $\Delta$ does not contain hubs and $\Theta$-edges, it is a diagram over the presentation of $\mathcal{K}$. Using the fact that $\mathcal{K}$ is a free product, we conclude that it is a diagram over $K^{(1)}$. But by Lemma 5.10. $X^{(1)} \cup Y^{(1)}$ freely generates a free subgroup in $K^{(1)}$. Hence the label of $\partial \Delta$ is freely trivial, and so $\mathcal{T}$ is not reduced, a contradiction.

Lemma 5.14. Suppose that $\Delta$ is a reduced diagram over $\mathcal{P}^{\prime}$ without $t_{j}$-edges on the boundary, $j=1, \ldots, N$. Suppose that $\Delta$ contains a hub. Then there exists a hub $\pi_{1}$ in $\Delta$ and an $i<N$ such that the $t_{i}$-band $\mathcal{T}_{1}$ and the $t_{i+1}$-band $\mathcal{T}_{2}$ starting on $\partial \pi_{1}$ end on the boundary of another hub $\pi_{2}$ of $\Delta$ and there are no hubs in the subdiagram bounded by the medians of $\mathcal{T}_{1}, \mathcal{T}_{2}$ and the parts of $\partial \pi_{1}, \partial \pi_{2}$ connecting the start edges of $\mathcal{T}_{1}, \mathcal{T}_{2}$ and not containing t-edges.

Proof. Indeed, since $\Delta$ does not contain $t_{j}$-edges on the boundary, it must contain at least two hubs. Consider the graph where vertices are hubs of $\Delta$ and edges are the $t_{j}$ bands connecting them. The boundary of a hub cell has label $W_{0}\left(\overline{\mathcal{S}}_{i}\right), i=1,2$, which has occurrences of $N$ letters $t_{1}, \ldots, t_{n}$. The corresponding edges on the boundary of the hub are start edges of $N$ t-bands connecting the hub with other hubs. Thus every vertex of this planar graph has degree $N \geq 12$. By Lemma 3.13, there must be two hubs connected by a $t_{i}$-and $t_{j}$-band, $i<j$. Therefore these two hubs are connected by a $t_{i}$-band and a $t_{i+1}$-band. Consider all subdiagrams $\Delta^{\prime}$ of $\Delta$ bounded by two hubs and two consecutive $t$-bands connecting them. Since the inside subdiagram of such a subdiagram does not contain $t_{j}$-edges on the boundary, a smallest area subdiagram $\Delta^{\prime}$ of this form will satisfy the conditions of the lemma. 
5.3. Sectors. Here we shall generalize Lemma 4.4.

Lemma 5.15. Suppose that a reduced diagram $\Delta$ over $\mathcal{P}^{\prime}$ without hubs has boundary

$$
\mathfrak{p q r}^{-1} \mathfrak{s}^{-1}
$$

where $\mathfrak{p}$ and $\mathfrak{r}$ are sides of $a t_{i}$ - and a $k_{i+1}$-band $\mathcal{T}_{1}, \mathcal{T}_{2}$, respectively, and $\operatorname{Lab}(\mathfrak{p})$ and $\operatorname{Lab}(\mathfrak{r})$ do not contain transition rules $\bar{\tau}_{1}^{ \pm 1}, \operatorname{Lab}(\mathfrak{q}) \equiv t_{i} W_{1} \bar{k}_{i+1}$ and $\operatorname{Lab}(\mathfrak{s}) \equiv$ $t_{i} W_{2} \bar{k}_{i+1}$ where $W_{1}, W_{2}$ are admissible words of $\mathcal{S}_{1}^{(2 i+1)}$ for some $i=1, \ldots, N-1$. Then the subdiagram of $\Delta$ bounded by the median of $\mathcal{T}_{1}$, the median of $\mathcal{T}_{2}, \mathfrak{q}, \mathfrak{s}$ is combinatorially $\mathcal{P}$-homotopic to a computational sector of $\mathcal{S}_{1}^{(2 i+1)}$.

Proof. By Lemma 5.12 every $\Theta$-band in $\Delta$ starts on $\mathfrak{p}$ or on $\mathfrak{r}$. Suppose that a $\Theta$ band starts and ends on $\mathfrak{p}$. Since $\Theta$-bands do not intersect, there exists a $\Theta$-band $\mathcal{A}$ whose start and end edges belong to the neighbor cells of $\mathcal{T}_{1}$. Then these cells cancel, a contradiction.

Similarly a $\Theta$-band cannot start and end on $\mathfrak{r}$. Therefore $\Theta$-bands that start on $\mathfrak{p}$ (on $\mathfrak{r}$ ) end on $\mathfrak{r}$ (on $\mathfrak{p}$ ). Let $\mathcal{A}_{1}, \mathcal{A}_{2}$ be two consecutive $\Theta$-bands in $\Delta$. These bands start on two consecutive $\Theta$-edges of $\mathfrak{p}$ and end on two consecutive $\Theta$-edges of $\mathfrak{r}$. The boundary of the subdiagram $\Delta^{\prime}$ bounded by the medians of $\mathcal{A}_{1}, \mathcal{A}_{2}$ and $\mathfrak{p}, \mathfrak{r}$ consists of edges with labels in $Z \cup Q$. The subdiagram $\Delta^{\prime}$ does not contain hubs and $\Theta$-edges, so it is a diagram over the presentation of $\mathcal{K}$. Therefore the boundary label of $\Delta^{\prime}$ is freely trivial, and $\Delta^{\prime}$ does not contain cells.

Hence all cells in $\Delta$ correspond to relations of $\overline{\mathcal{S}}_{1}$. It remains to use Lemma 4.4 .

The following lemma is proved in the same way as Lemma 5.15

Lemma 5.16. Suppose that a reduced diagram $\Delta$ over $\mathcal{P}^{\prime}$ without hubs has boundary

$$
\mathfrak{p q r}^{-1} \mathfrak{s}^{-1}
$$

where $\mathfrak{p}$ and $\mathfrak{r}$ are sides of a $t_{i}$ - and a $k_{i+1}$-band $\mathcal{T}_{1}, \mathcal{T}_{2}$, respectively, and Lab( $\left.\mathfrak{p}\right)$ and $\operatorname{Lab}(\mathfrak{r})$ do not contain transition rules $\bar{\tau}_{2}^{ \pm 1}, \operatorname{Lab}(\mathfrak{q}) \equiv t_{i} W_{1} \hat{k}_{i+1}$ and $\operatorname{Lab}(\mathfrak{s}) \equiv$ $t_{i} W_{2} \hat{k}_{i+1}$ where $W_{1}, W_{2}$ are admissible words of $\mathcal{S}_{2}^{(2 i+1)}$ for some $i=1, \ldots, N-1$. Then the subdiagram of $\Delta$ bounded by the median of $\mathcal{T}_{1}$, the median of $\mathcal{T}_{2}, \mathfrak{q}, \mathfrak{s}$ is combinatorially $\mathcal{P}$-homotopic to a computational sector of $\mathcal{S}_{2}^{(2 i+1)}$.

\subsection{Diagrams with boundary label over $\varepsilon(A)$.}

Lemma 5.17. Let $\Delta$ be a diagram over $\mathcal{P}^{\prime}$ without hubs. Then it does not contain $\left(\Theta, k_{i}\right)$-annuli, $N>i \geq 1$.

Proof. Suppose that there exists a $(\Theta, k)$-annulus $\mathcal{T}$ in $\Delta$ composed of a $\Theta$-band $\mathcal{A}$ and a $k$-band $\mathcal{B}$. Since $\Theta$-bands do not intersect, we can assume that the inside diagram $\Delta^{\prime}$ of $\mathcal{T}$ does not have $\Theta$-edges (by Lemma 5.12). Therefore $\Delta^{\prime}$ is a diagram over the presentation of the group $\mathcal{K}$.

Suppose that $\Delta^{\prime}$ contains $k_{j}$-edges. Then it contains a $k_{j}$-annulus for some $j$. By Lemma 5.13, $j>1$. Consider an innermost such annulus. The reduced boundary label of its inside diagram is a word in $X^{(s)} \cup Y^{(s)}$ for some $s>1$. But $X^{(s)} \cup Y^{(s)}$ freely generates a free subgroup of $\mathcal{K}$ by Lemma 3.3. so that annulus contains two cells that cancel. Hence $\Delta^{\prime}$ does not contain $k_{j}$-edges. 
Therefore $\Delta^{\prime}$ is a diagram over the presentation of one of the $H(A)^{(s)}, s>1$. The label of the boundary of $\Delta^{\prime}$ is then a product $U V$ where $U$ is a word in $A^{(i)} \cup B^{(i)}$ and $V$ is a word in $X^{(i)} \cup Y^{(i)}$. By Lemma 3.3, $U=V=1$ in the free group. Hence either $\mathcal{A}$ or $\mathcal{B}$ contains two cells that cancel, a contradiction.

Lemma 5.18. Let $\Delta$ be a reduced diagram over the presentation $\mathcal{P}^{\prime}$ with $\partial \Delta$ consisting of $\varepsilon(A)$-edges (that includes the case when $\Delta$ is spherical). Suppose that $\Delta$ does not contain hubs. Then $\Delta$ is combinatorially $\mathcal{P}$-homotopic to a diagram consisting of $\Gamma$-cells.

Proof. Suppose that $\Delta$ contains $\Theta$-edges. Then by Lemma $5.12, \partial \Delta$ contains $\Theta$ edges, a contradiction. Hence all cells in $\Delta$ correspond to the relations of $\mathcal{K}$. Since $\mathcal{K}$ is a free product of $K^{(i)}, i=1, \ldots, N$, and the boundary label is in $K^{(1)}$, all cells in $\Delta$ correspond to relations of $K^{(1)}$ (consider a maximal subdiagram consisting of cells corresponding to relations of other free factors; all the boundary components of that subdiagram must be empty). By Lemma 5.13. $\Delta$ does not contain $k_{1}$-edges. Hence $\Delta$ is a diagram over the presentation of the free product of $K_{1}^{(1)}$ and $K_{2}^{(1)}$. Since the boundary label in $\Delta$ is from $K_{1}^{(1)}$, all cells in $\Delta$ correspond to relations of $K_{1}^{(1)}$ which is a copy of $H^{\prime}(\Gamma)$. It remains to use the first statement of Lemma 3.21 .

A not necessarily reduced diagram over $\mathcal{P}^{\prime}$ is called normal if every hub is contained in a standard subdiagram (i.e. subdiagram of the form $\Psi(r), r \in R$; see Notation 5.5), standard subdiagrams do not share cells, and every 2 -hub $i$-subdiagram is reduced, $i<N$.

Lemma 5.19. Let $1 \leq i<N$. Then every reduced 2 -hub $i$-diagram $\Delta$ (see Notation 5.5) over $\mathcal{P}^{\prime}$ is combinatorially $\mathcal{P}$-homotopic to a normal diagram.

Proof. Suppose first that the complexity of $\Delta$ is 0 . Then both hubs in $\Delta$ are first hubs or both of them are second hubs. Assume both are first hubs. By Lemma 5.15. the subdiagram of $\Delta$ bounded by the medians of $\mathcal{X}_{1}, \mathcal{X}_{2}$ and parts of the boundaries of the hubs (and not containing the hubs) is combinatorially homotopic to a computational sector of $\mathcal{S}_{1}^{(2 i+1)}$. By Proposition 4.2(4), that computation is empty, so $\mathcal{T}_{1}$ and $\mathcal{T}_{2}$ are empty, and the two hubs in $\Delta$ cancel, a contradiction. The case when both hubs are second hubs is similar except that one needs to use Lemma 5.16 instead of 5.15

Suppose now that $\Delta$ has complexity $\geq 1$. Let $\mathcal{A}_{1}$ be the first transition $\Theta$-band in $\Delta$ counting from $\pi_{1}$ to $\pi_{2}$.

Case 1. Let us assume that $\pi_{1}$ is the first hub.

Then $\mathcal{A}_{1}$ must be a $\bar{\tau}_{1}$-band (since the letter in $\operatorname{Lab}\left(\pi_{1}\right)$ from $\left\{\tilde{k}_{i+1}, k_{i+1}, \hat{k}_{i+1}\right\}$ is $\tilde{k}_{i+1}$ and relations involving $\bar{\tau}_{2}$ do not contain that letter). The side of $\mathcal{A}_{1}$ that is further from $\pi_{1}$ must contain $k_{i+1}$. Hence the $\Theta$-band $\mathcal{A}_{2}$ which is next after $\mathcal{A}_{1}$ in $\Delta$ (counting from $\pi_{1}$ to $\pi_{2}$ ) must be either a $\bar{\tau}_{1}^{-1}$-band or $\bar{\tau}_{2}^{-1}$-band. The first option is impossible because $\mathcal{A}_{1}$ and $\mathcal{A}_{2}$ intersect $\mathcal{T}_{1}$ in two neighbor cells, and if the first option occurs, these cells cancel, which would contradict the assumption that $\Delta$ is reduced. Thus $\mathcal{A}_{2}$ is a $\bar{\tau}_{2}^{-1}$-band.

The medians of $\mathcal{A}_{1}$ and $\mathcal{A}_{2}$ cut the diagram $\Delta$ into three parts (see the top part of Figure 25]: $\Delta_{1}, \Delta_{2}, \Delta_{3}$ where $\Delta_{1}$ contains $\pi_{1}, \Delta_{3}$ contains $\pi_{2}$. Let $\Delta_{1}^{\prime}$ be the diagram $\Delta_{1}$ without the hub cell. Note that the boundary of $\Delta_{1}^{\prime}$ has the form 

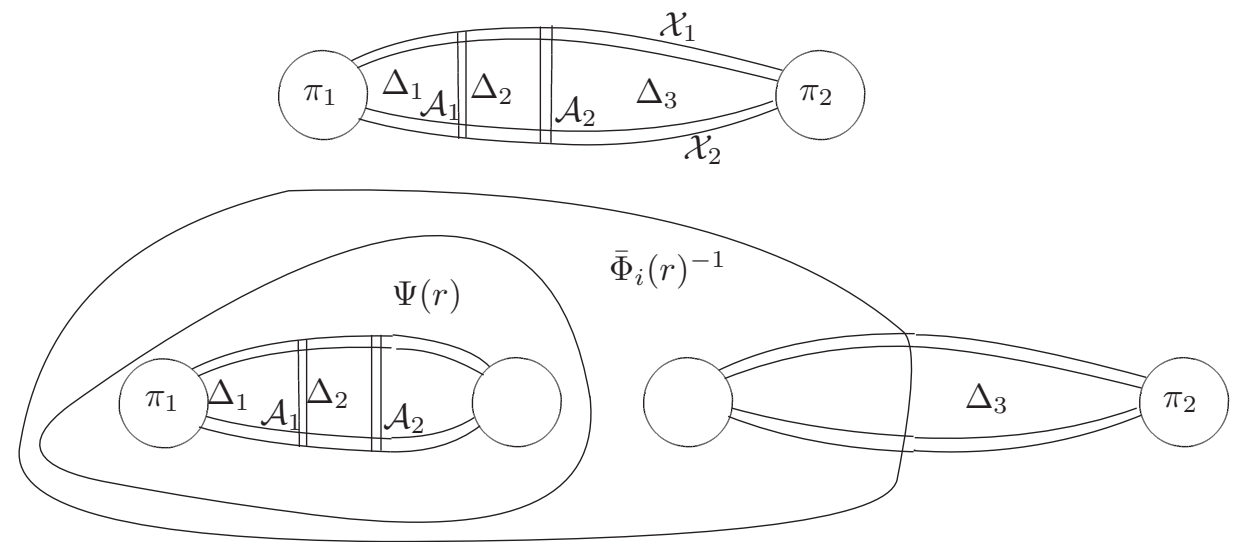

FiguRE 25

$\mathfrak{p q r}^{-1} \mathfrak{s}^{-1}$ where $\mathfrak{p}, \mathfrak{r}$ are sides of a $t_{i^{-}}$and $k_{i+1^{-}}$-band, respectively, not containing transition cells, $\operatorname{Lab}(\mathfrak{q}) \equiv W_{0}\left(\mathcal{S}_{1}^{(2 i+1)}\right)$,

$$
\operatorname{Lab}(s) \equiv t_{i} \tilde{q}_{1}^{(2 i+1)} \tilde{q}_{2}^{(2 i+1)} U \tilde{q}_{3}^{(2 i+1)} \ldots \tilde{q}_{m}^{(2 i+1)} \tilde{k}_{i+1}
$$

where $U$ is a word in $Z_{2}^{(2 i+1)}$. By Lemma 5.15, that diagram without the $t_{i}$-band and the $k_{i+1}$-band is combinatorially $\mathcal{P}$-homotopic to a computational sector corresponding to a computation of $\mathcal{S}_{1}^{(2 i+1)}$ accepting the word $\tilde{q}_{1}^{(2 i+1)} \tilde{q}_{2}^{(2 i+1)} U \tilde{q}_{3}^{(2 i+1)} \ldots$ $\tilde{q}_{m}^{(2 i+1)}$. By the choice of the $S$-machine $\mathcal{S}_{1}$, then $U$ is a copy of $U(r)$ for some $r \in R$, and by Lemma $4.2(3)$, the diagram $\Delta_{1}$ is uniquely determined (up to $\mathcal{P}^{\prime}$-homotopy) by $r$.

The $k_{i+1}$-band in $\Delta_{2}$ consists of gluing $k_{i+1}$-cells. Indeed, no $\left(\Theta, k_{i+1}\right)$-relation except transition cells involves letter $k_{i+1}$ (they involve letters $\tilde{k}_{i+1}$ and $\hat{k}_{i+1}$ ) and the $k_{i+1}$-band in $\Delta_{2}$ does not contain $\Theta$-edges by Lemma 5.17 .

Let $\Delta_{2}^{\prime}$ be the diagram obtained from $\Delta_{2}$ by removing the $k_{i+1}$-band and the maximal $q_{2}^{(2 i+1)}$-band started on $\partial \Delta_{2}$. By Lemma 5.17 that $k_{i+1}$-band does not contain $\Theta$-edges. Since the intersections of $\mathcal{A}_{1}, \mathcal{A}_{2}$ with the $t_{i}$-band $\mathcal{X}_{1}$ (resp. with the maximal $Q_{1}^{(2 i+1)}$-band starting on $\left.\partial \Delta_{2}\right)$ are neighbor cells on this band, the boundary of diagram $\Delta_{2}^{\prime}$ has the form $\mathfrak{q}_{1} \mathfrak{r}_{1}^{-1} \mathfrak{s}^{-1}$ where $\operatorname{Lab}\left(\mathfrak{q}_{1}\right)=U(r), \operatorname{Lab}\left(\mathfrak{r}_{1}\right)$ is a word in $X^{(2 i+1)} \cup Y^{(2 i+1)}, \operatorname{Lab}(s)$ is a product of words of the form $\mu(a)^{(2 i+1)}$, $a \in A$. By Lemma 3.17, the diagram $\Delta_{2}^{\prime}$ is determined by the word $U(r)$ up to combinatorial $\mathcal{P}$-homotopy. Therefore the diagram $\Delta_{2}$ is determined by $r$ up to combinatorial $\mathcal{P}$-homotopy (and is homotopic to $\Upsilon(\mu(r))$ ).

Case 2. Now assume that $\pi_{1}$ is the second hub.

Then $\mathcal{A}_{1}$ must be a $\bar{\tau}_{2}$-band (since the letter in $\operatorname{Lab}\left(\pi_{1}\right)$ from $\left\{\tilde{k}_{i+1}, k_{i+1}, \hat{k}_{i+1}\right\}$ is $\hat{k}_{i+1}$ and relations involving $\bar{\tau}_{1}$ do not contain that letter). The side of $\mathcal{A}_{1}$ that is further from $\pi_{1}$ must contain $k_{i+1}$. Similarly to the previous case, the next after $\mathcal{A}_{1} \Theta$-band $\mathcal{A}_{2}$ in $\Delta$ (counting from $\pi_{1}$ to $\pi_{2}$ ) is a $\bar{\tau}_{1}^{-1}$-band.

As in Case 1 , the medians of $\mathcal{A}_{1}$ and $\mathcal{A}_{2}$ cut the diagram $\Delta$ into three parts $\Delta_{1}, \Delta_{2}, \Delta_{3}$ where $\Delta_{1}$ contains $\pi_{1}, \Delta_{3}$ contains $\pi_{2}$. Let $\Delta_{1}^{\prime}$ be the diagram $\Delta_{1}$ without the hub cell. Note that the boundary of $\Delta_{1}^{\prime}$ has the form $\mathfrak{p q r}^{-1} \mathfrak{s}^{-1}$ where 
$\mathfrak{p}, \mathfrak{r}$ are sides of a $t_{i^{-}}$and $k_{i+1}$-band, respectively, not containing transition cells, $\operatorname{Lab}(\mathfrak{q}) \equiv W_{0}\left(\mathcal{S}_{1}^{(2 i+1)}\right)$,

$$
\operatorname{Lab}(s) \equiv t_{i} \tilde{q}_{1}^{(2 i+1)} \tilde{q}_{2}^{(2 i+1)} U \tilde{q}_{3}^{(2 i+1)} \ldots \tilde{q}_{m}^{(2 i+1)} \tilde{k}_{i+1}
$$

where $U$ is a word in $Z_{2}^{(2 i+1)}$. By Lemma 5.16, that diagram without the $t_{i}$-band and the $k_{i+1}$-band is combinatorially $\mathcal{P}$-homotopic to a computational sector corresponding to a computation of $\mathcal{S}_{2}^{(2 i+1)}$ accepting the word $\hat{q}_{1}^{(2 i+1)} U \hat{q}_{2}^{(2 i+1)} \hat{q}_{3}^{(2 i+1)} \ldots$ $\hat{q}_{m}^{(2 i+1)}$. By the choice of the $S$-machine $\mathcal{S}_{2}$, then $U \equiv \varepsilon(r)$ for some $r \in R$, and by Lemma 4.2(3), the diagram $\Delta_{1}$ is uniquely determined (up to $\mathcal{P}^{\prime}$-homotopy) by $r$.

As in Case 1 , the $k_{i+1}$-band in $\Delta_{2}$ consists of gluing $k_{i+1}$-cells. Let $\Delta_{2}^{\prime}$ be the diagram obtained from $\Delta_{2}$ by removing the $k_{i+1}$-band and the maximal $q_{2}^{(2 i+1)}$ band started on $\partial \Delta_{2}$. By Lemma 5.17 that $k_{i+1}$-band does not contain $\Theta$-edges. Since the intersections of $\mathcal{A}_{1}, \mathcal{A}_{2}$ with the $t_{i}$-band $\mathcal{X}_{1}$ (resp. with the maximal $Q_{1}^{(2 i+1)}$-band starting on $\partial \Delta_{2}$ ) are neighbor cells on this band, the boundary of diagram $\Delta_{2}^{\prime}$ has the form $\mathfrak{q}_{1} \mathfrak{r}_{1}^{-1} \mathfrak{s}^{-1}$ where $\operatorname{Lab}\left(\mathfrak{q}_{1}\right)=U^{\prime}, \operatorname{Lab}\left(\mathfrak{r}_{1}\right)$ is a word in $X^{(2 i+1)} \cup Y^{(2 i+1)}, \operatorname{Lab}(s)=\mu(r)$. By Lemma 3.17 the diagram $\Delta_{2}^{\prime}$ is determined by the word $\mu(r)$ up to combinatorial $\mathcal{P}$-homotopy. Therefore the diagram $\Delta_{2}$ is determined by $r$ up to combinatorial $\mathcal{P}$-homotopy (and is homotopic to $\Upsilon(\mu(r))$ ).

Thus in both cases we have proved that up to combinatorial homotopy the subdiagram $\Delta^{\prime \prime}=\Delta_{1} \cup \mathcal{A}_{1} \cup \Delta_{2} \cup \mathcal{A}_{2}$ of $\Delta$ coincides with the corresponding subdiagram $\Phi_{i}(r)$ of the diagram $\Psi(r)$ described in Notation 5.3

Since diagrams $\Delta$ and $\Psi_{i}(r)$ have a common subdiagram $\Delta^{\prime \prime}$, we can apply the standard surgery described in Section 2.1.4 we cut $\Delta$ along the boundary of $\Delta^{\prime \prime}$, insert into the resulting hole the diagram $\bar{\Phi}_{i}(r)$ (the complement of $\Phi_{i}(r)$ in $\Psi(r)$; see Notation 5.3) and its mirror image $\bar{\Phi}_{1}(r)^{-1}$ so that $\Delta^{\prime \prime} \cup \bar{\Phi}_{i}(r)$ is combinatorially $\mathcal{P}$-homotopic to $\Psi(r)$, and the subdiagram $\bar{\Phi}_{i}(r)$ and its mirror image $\bar{\Phi}_{i}(r)^{-1}$ cancel each other. The new diagram $\breve{\Delta}$, after a combinatorial $\mathcal{P}$-homotopy, becomes a union of a subdiagram that is a copy of $\Psi(r)$, a 2-hub reduced $i$-subdiagram of smaller complexity than $\Delta$, and a number of non-hub cells corresponding to relations of $\mathcal{P}$ (see the bottom part of Figure 25). This allows us to proceed by induction on the complexity of the 2-hub $i$-subdiagram.

Lemma 5.20. Let $\Delta$ be any reduced diagram over $\mathcal{P}$ whose boundary label is a word in $\varepsilon(A)$. Then $\Delta$ is combinatorially $\mathcal{P}$-homotopic to a normal diagram that coincides with the union of its standard subdiagrams.

Proof. Every normal diagram over $\mathcal{P}$ can be viewed as a diagram over $\mathcal{P}^{\prime}$ : ignore the cells of standard subdiagrams and view these subdiagrams as $\Gamma$-cells (the standard diagram $\Psi(r)$ has boundary label $\varepsilon(r)$ by Notation 5.3). Therefore if $\Delta$ does not have hubs, we can apply Lemma 5.18. The combinatorial $\mathcal{P}$-homotopy from Lemma 5.18 does not touch the $\Gamma$-cells (since $\mathcal{P}$ does not contain $\Gamma$-relations), so $\Delta$ is combinatorially $\mathcal{P}$-homotopic to a normal diagram.

Suppose that $\Delta$ has hubs. Consider the graph $\nabla=\nabla(\Delta)$ whose vertices are the hubs of $\Delta$. Two hubs are adjacent if there exists a $t_{i}$-band, $i<N$, connecting them. Since no vertex is adjacent to itself and the graph is planar $\left(t_{i}\right.$-bands do not intersect), we apply Lemma 3.13 again and conclude that $\nabla$ has a vertex of degree 5 . Since $\partial \Delta$ does not have $t_{i}$-edges, there exist two hubs $\pi_{1}, \pi_{2}$ connected by a $t_{i}$-band and a $t_{j}$-band, $i<j<N$ (recall that $N \geq 12$ ). Consider the subdiagram 
$\Delta^{\prime}$ of $\Delta$ bounded by the two hubs $\pi_{1}, \pi_{2}$ and the $t_{i^{-}}$and $t_{j}$-band. Assume that $\Delta^{\prime}$ is a smallest under inclusion such subdiagram of $\Delta$. Suppose that $\Delta^{\prime}$ contains hubs. Then the graph $\nabla\left(\Delta^{\prime}\right)$ contains a vertex of degree 5 . By the minimality of $\Delta^{\prime}$, no two hubs of $\Delta^{\prime}$ are connected by a $t_{i^{\prime}}$ and $t_{j^{\prime}}$-band, $i^{\prime}<j^{\prime}<N$. Hence there exists a hub $\pi_{3} \in \Delta^{\prime}$ which has at least three of its $t_{j}$-bands, $j<N$, each connecting that hub with $\pi_{1}$ or $\pi_{2}$ (by the pigeon-hole property, since $11>2 \times 5$ ). Then either $\pi_{1}$ or $\pi_{2}$ is connected with $\pi_{3}$ by two of these bands, which contradicts the minimality of $\Delta^{\prime}$. Hence we can assume that $\Delta^{\prime}$ does not have hubs. Then the $k_{i+1}$-band starting on $\partial \pi_{1}$ ends on $\partial \pi_{2}$. Consider the subdiagram $\Delta^{\prime \prime}$ bounded by $\pi_{1}, \pi_{2}$, the $t_{i}$-band and the $k_{i+1}$-band connecting them. Viewed as a diagram over $\mathcal{P}^{\prime}$ (we again consider standard subdiagrams as $\Gamma$-cells) $\Delta^{\prime \prime}$ is a 2 -hub $i$-subdiagram of $\Delta$. By Lemma 5.19, this subdiagram is combinatorially $\mathcal{P}$-homotopic to a normal diagram. Replacing $\Delta^{\prime \prime}$ by that normal diagram, we obtain a diagram with the same boundary label as $\Delta$ but with fewer hubs outside standard subdiagrams (note that this homotopy does not touch cells outside $\Delta^{\prime \prime}$ ), so we can proceed by induction on the number of hubs outside standard subdiagrams.

The following two lemmas complete the proof of Theorem 5.1

Lemma 5.21 (Part (2) of Theorem 5.1). The map $\varepsilon: \Gamma \rightarrow \mathcal{G}$ is injective.

Proof. Suppose that for some word $u$ in $A, \varepsilon(u)=1$ in $\mathcal{G}$. Then there exists a van Kampen diagram $\Delta$ over $\mathcal{P}$ with boundary label $\varepsilon(u)$. By Lemma 5.20, $\Delta$ is combinatorially homotopic to a normal diagram tesselated by standard subdiagrams. If we view each of these subdiagrams as a $\Gamma$-cell, $\Delta$ becomes a diagram $\Delta_{1}$ over the presentation $\langle\varepsilon(A) \mid \varepsilon(R)\rangle$. Applying $\varepsilon^{-1}$ to the labels of $\Delta_{1}$, we obtain a van Kampen diagram over the presentation of $\Gamma$ with boundary label $u$. Hence $u=1$ in $\Gamma$.

Lemma 5.22 (Part (3) of Theorem 5.1). If the presentation $\langle A \mid R\rangle$ of $\Gamma$ is combinatorially aspherical, then the presentation $\mathcal{P}$ of $\mathcal{G}$ is combinatorially aspherical.

Proof. Consider a spherical diagram $\Delta$ over $\mathcal{P}$. It is a disc diagram with empty boundary. By Lemma 5.20, $\Delta$ is combinatorially $\mathcal{P}$-homotopic to a spherical normal diagram $\Delta_{1}$ tesselated by standard subdiagrams. Again, view $\Delta_{1}$ as a spherical diagram over the presentation of $\Gamma$. Since that presentation is aspherical by our assumption, $\Delta_{1}$ can be combinatorially deformed to a trivial diagram by diamond moves and insertions and deletions of cancelable standard subdiagrams. Therefore $\Delta$ is combinatorially homotopic to a trivial diagram.

Remark 5.23. It is possible to show, using the ideas from [2], that the embedding constructed in this paper is quasi-isometric if we choose the number $N$ large enough (i.e. the subgroup $\varepsilon(\Gamma)$ embeds without distortion) and preserves solvability of the word problem. But the Dehn function of the finitely presented group $\mathcal{G}$ is almost always superexponential: indeed, by Remark 3.7. the computational sector of $\mathcal{S}_{1}$ for the computation accepting $U(r)$ has superexponential area in terms of $|r|$ and is a subdiagram in the van Kampen diagram for the relation $\varepsilon(r)=1$ in $\mathcal{G}$. D. Osin asked whether one can embed every recursively presented group into a finitely presented group as a malnormal subgroup. It is possible to prove that $\varepsilon(\Gamma) \mathrm{em}-$ beds malnormally (regardless of whether $\Gamma$ is aspherical or not), so the answer to that question is affirmative. That will be proved in our next paper. It would be 
interesting to find out whether our construction preserves several other properties including the finite decomposition complexity of Guentner, Tessera, and Yu 13. Also notice that our Corollary 1.2 provides the only known potential counterexample to various versions of the Novikov and the Baum-Connes conjectures. It would be interesting of course to find out if these are really counterexamples.

\section{ACKNowledgements}

This paper completes a project started about thirteen years ago after M. Gromov first announced the construction of his random finitely generated groups containing expanders and a question of whether these groups embed into groups with finite $K(., 1)$ arose. During these years, the author was helped by several people including D. Osin and E. Rips. The author is especially grateful to A. Yu. Ol'shanskiu for many useful discussions.

\section{REFERENCES}

[1] G. N. Arzhantseva, T. Delzant, Examples of random groups, preprint, 2008.

[2] J.-C. Birget, A. Yu. Ol'shanskii, E. Rips, and M. V. Sapir, Isoperimetric functions of groups and computational complexity of the word problem, Ann. of Math. (2) 156 (2002), no. 2, 467-518, DOI 10.2307/3597196. MR1933724(2005b:20077b)

[3] G. Baumslag, E. Dyer, and C. F. Miller III, On the integral homology of finitely presented groups, Topology 22 (1983), no. 1, 27-46, DOI 10.1016/0040-9383(83)90044-7. MR682058 (85g:20044)

[4] Oleg Bogopolski and Enric Ventura, A recursive presentation for Mihailova's subgroup, Groups Geom. Dyn. 4 (2010), no. 3, 407-417, DOI 10.4171/GGD/88. MR2653968 (2011f:20072)

[5] Ian M. Chiswell, Donald J. Collins, and Johannes Huebschmann, Aspherical group presentations, Math. Z. 178 (1981), no. 1, 1-36, DOI 10.1007/BF01218369. MR627092 (83a:20046)

[6] D. J. Collins and J. Huebschmann, Spherical diagrams and identities among relations, Math. Ann. 261 (1982), no. 2, 155-183, DOI 10.1007/BF01456216. MR675732 (84b:20035)

[7] Rémi Coulon, Small Cancellation Theory and Burnside problem, preprint, arXiv:1302.6933, to appear in IJAC.

[8] Michael W. Davis, Exotic aspherical manifolds, Topology of high-dimensional manifolds, No. 1, 2 (Trieste, 2001), ICTP Lect. Notes, vol. 9, Abdus Salam Int. Cent. Theoret. Phys., Trieste, 2002, pp. 371-404. MR 1937019 (2004a:57031)

[9] Michael W. Davis, The geometry and topology of Coxeter groups, London Mathematical Society Monographs Series, vol. 32, Princeton University Press, Princeton, NJ, 2008. MR2360474 (2008k:20091)

[10] Alexander Dranishnikov, Open problems in asymptotic dimension theory, preprint, http://www.aimath.org/pggt/Asymptotic_Dimension, 2008.

[11] M. Gromov, Asymptotic invariants of infinite groups, Geometric group theory, Vol. 2 (Sussex, 1991), London Math. Soc. Lecture Note Ser., vol. 182, Cambridge Univ. Press, Cambridge, 1993, pp. 1-295. MR1253544 (95m:20041)

[12] M. Gromov, Random walk in random groups, Geom. Funct. Anal. 13 (2003), no. 1, 73-146, DOI 10.1007/s000390300002. MR 1978492(2004j:20088a)

[13] Erik Guentner, Romain Tessera, and Guoliang Yu, A notion of geometric complexity and its application to topological rigidity, Invent. Math. 189 (2012), no. 2, 315-357, DOI 10.1007/s00222-011-0366-z. MR2947546

[14] P. J. Heawood, Map-Colour Theorems, Quarterly Journal of Mathematics, Oxford 24, 1890, 332-338.

[15] G. Higman, Subgroups of finitely presented groups, Proc. Roy. Soc. Ser. A 262 (1961), 455475. MR0130286 (24 \#A152)

[16] N. Higson, V. Lafforgue, and G. Skandalis, Counterexamples to the Baum-Connes conjecture, Geom. Funct. Anal. 12 (2002), no. 2, 330-354, DOI 10.1007/s00039-002-8249-5. MR1911663 (2003g:19007) 
[17] Wolfgang Lück, Survey on aspherical manifolds, European Congress of Mathematics, Eur. Math. Soc., Zürich, 2010, pp. 53-82, DOI 10.4171/077-1/4. MR2648321 (2011d:57061)

[18] Roger C. Lyndon and Paul E. Schupp, Combinatorial group theory, Springer-Verlag, Berlin, 1977. Ergebnisse der Mathematik und ihrer Grenzgebiete, Band 89. MR0577064 (58 \#28182)

[19] Alexey Muranov, Finitely generated infinite simple groups of infinite square width and vanishing stable commutator length, J. Topol. Anal. 2 (2010), no. 3, 341-384, DOI 10.1142/S1793525310000380. MR2718128 (2011i:20052)

[20] A. Yu. Ol'shanskiı̌, Geometry of defining relations in groups, Mathematics and its Applications (Soviet Series), vol. 70, Kluwer Academic Publishers Group, Dordrecht, 1991. Translated from the 1989 Russian original by Yu. A. Bakhturin. MR:1191619 (93g:20071)

[21] A. Yu. Ol'shanskiı̌, SQ-universality of hyperbolic groups, Mat. Sb. 186 (1995), no. 8, 119132, DOI 10.1070/SM1995v186n08ABEH000063 (Russian, with Russian summary); English transl., Sb. Math. 186 (1995), no. 8, 1199-1211. MR1357360 (97b:20057)

[22] Alexander Yu. Ol'shanskii, Denis V. Osin, and Mark V. Sapir, Lacunary hyperbolic groups, Geom. Topol. 13 (2009), no. 4, 2051-2140, DOI 10.2140/gt.2009.13.2051. With an appendix by Michael Kapovich and Bruce Kleiner. MR2507115 (2010i:20045)

[23] A. Yu. Ol'shanskii and M. V. Sapir, The conjugacy problem and Higman embeddings, Mem. Amer. Math. Soc. 170 (2004), no. 804, viii+133. MR2052958 (2005f:20062)

[24] A. Yu. Ol'shanskii and M. V. Sapir, Groups with small Dehn functions and bipartite chord diagrams, Geom. Funct. Anal. 16 (2006), no. 6, 1324-1376, DOI 10.1007/s00039-006-0580-9. MR.2276542(2008k:20053)

[25] Joseph J. Rotman, An introduction to the theory of groups, 4th ed., Graduate Texts in Mathematics, vol. 148, Springer-Verlag, New York, 1995. MR1307623 (95m:20001)

[26] Mark Sapir, Algorithmic and asymptotic properties of groups, International Congress of Mathematicians. Vol. II, Eur. Math. Soc., Zürich, 2006, pp. 223-244. MR2275595 (2008f:20105)

[27] Mark Sapir, Asymptotic invariants, complexity of groups and related problems, Bull. Math. Sci. 1 (2011), no. 2, 277-364, DOI 10.1007/s13373-011-0008-1. MR2901003

[28] Mark Sapir, Non-commutative combinatorial algebra, Springer, to appear, 2014.

[29] Mark V. Sapir, Jean-Camille Birget, and Eliyahu Rips, Isoperimetric and isodiametric functions of groups, Ann. of Math. (2) 156 (2002), no. 2, 345-466, DOI 10.2307/3597195. MR 1933723 (2005b:20077a)

[30] Rufus Willett, Property A and graphs with large girth, J. Topol. Anal. 3 (2011), no. 3, 377384, DOI 10.1142/S179352531100057X. MR2831267 (2012j:53047)

[31] Guoliang Yu, The Novikov conjecture for groups with finite asymptotic dimension, Ann. of Math. (2) 147 (1998), no. 2, 325-355, DOI 10.2307/121011. MR:1626745 (99k:57072)

[32] Guoliang Yu, The coarse Baum-Connes conjecture for spaces which admit a uniform embedding into Hilbert space, Invent. Math. 139 (2000), no. 1, 201-240, DOI 10.1007/s002229900032. MR 1728880(2000j:19005)

Department of Mathematics, Vanderbilt University, Nashville, Tennessee 37240

E-mail address: m.sapir@vanderbilt.edu 